\title{
Deformation behavior of nanocomposites studied by X-ray scattering: Instrumentation and methodology
}

\author{
Norbert Stribeck
}

\section{Introduction}

Overview. X-ray scattering from polymer composites during deformation has not yet become a routine method. Thus, a scientific group that intends to be successful in this field, still has to provide developer's qualifications both in the fields of engineering and computer programming. For this reason this chapter does not focus on the documentation of results, but instead on the presentation of the state of development, of practical guidelines as an aid to avoid errors, and of developing strategies for a broader application of this method.

Studies of deformation by X-ray scattering are presently experiencing a change from the socalled stretch-hold ${ }^{1}$ technique [1] to an in situ study of nanostructure evolution during dynamical tensile tests. Thus, here the new perspectives, their technical foundations, and first results are discussed in comparison to those of earlier studies. Ultimately, such studies are carried out in order to answer questions of some practical relevance: What is the constitution of the nanostructure of the composite that imprints tailored properties to the material like special toughness or low fatigue? Such new materials are urgently sought after e.g. in automotive industry, in order to become able to accomplish the politically requested goals of climate protection in the near future by reduction of weight.

As research politics is presently urging science to force the pace by utilizing computer modelling methods, the theoretician who is up to develop the model needs an accurate description of the complex nanostructure and understanding of the important evolution mechanisms. Section 6 presents an example which demonstrates that modelling the mechanical properties of such a simple material like polypropylene requires consideration of strain induced crystallization and relaxation induced melting. Unfortunately, to my best knowledge, the modelling of such complexity is still beyond the development status of the computer modelling method. Thus, the practical modelling of the mechanics of a polymer nanocomposite is a huge challenge.

From statical to dynamical experiments. The investigation of deformation by the smallangle X-ray scattering (SAXS) method has been in a dilemma for decades. On the one hand, the $\mathrm{X}$-ray method is promising to be able to reveal the structure of the material in situ, on the other

\footnotetext{
${ }^{1}$ In the stretch-hold technique the sample is first stretched to a desired elongation and, second, an X-ray scattering pattern is taken in the hold state.
} 
hand, a deformation study implies that, in general, two-dimensional (2D) scattering patterns must be recorded. Until recently one had to choose either to record noisy patterns while the mechanical test was dynamically performed, or to carry out the test step-wise, which is far from reality. Only by means of the last mentioned technique it was possible to expose the material for a sufficiently long time at various constant elongations ("stretch-hold technique").

Noisy patterns are only appropriate for a qualitative interpretation of the structural changes in the material, which is afflicted with considerable uncertainties. In order to perform a quantitative analysis of the nanostructure, clear patterns are required. Nevertheless, if these clear images are originating from an experiment that carried out in a manner which is far from a realistic loading of the material, it remains highly questionable, how far the obtained results can be transferred to the behavior of the material during daily use.

Enhancement of the strain rate. Scientists have only been able for a short time to record good SAXS patterns with a cycle time of $30 \mathrm{~s}$ even from thin samples at synchrotron beamlines that are easily accessible ${ }^{2}$. Exploiting such short cycle times at slow strain rates $\left(\dot{\varepsilon} \approx 10^{-3} \mathrm{~s}^{-1}\right)$ it is possible to monitor a dynamic mechanical test by SAXS measurements which are sufficiently accurate to study the nanostructure evolution inside the material. Admittedly, such strain rates are still too low, but the technological gap is narrowing. By the time when the number of 3rd generation synchrotron sources has grown and the newly advertised high-power detectors will be available for materials research, the cycle time should decrease by a factor of 30 to $1 \mathrm{~s}$. Reciprocally, the maximum $\dot{\varepsilon}$ will increase. At HASYLAB, Hamburg in Germany corresponding experiments will be enabled beginning from fall 2008.

If noisy scattering patterns are accepted, even today mechanical tests performed at strain rates of practical relevance can be performed. This has been shown at the SRS Daresbury, Great Britain, where since 1995 structural evolution is studied at cycle times down to $40 \mathrm{~ms}$ [2-5].

Adapted extensometers and fatigue testing. It is still impossible or difficult to mount commercial tensile testers ${ }^{3}$ at a synchrotron beamline, and for stretch-hold experiments even very simple designs are often sufficient. Only the perspective of genuine in situ experiments initiated the construction of extensometers adapted for operation in synchrotron beamlines with all the options of commercial devices. These options comprise the automatic recording of stress and strain to be assigned to the respective scattering patterns, and the possibility to run complex test programs.

At this stage of development even a realization of fatigue tests comes into reach. The corresponding load-reversal experiments can be carried out, after the corresponding control programs for the mechanical tester have been written. From the results of such experiments one should be able to deduce, how the topology of the nanocomposite is changing during the fatigue test. Thus, one should gain access to a deeper understanding of the mechanisms of materials fatigue.

Literature at a glance. Static investigations of strained polymer materials are, in fact, the earliest demonstrations of the SAXS method [7-9]. To this day fibers and other nanocomposite materials are subjected to mechanical tests and investigated by X-ray scattering. Results of such

\footnotetext{
${ }^{2}$ For this purpose even a 2 nd generation synchrotron is sufficient, if optimized beamline optics, a modern detector, and advanced data processing are combined. To my experience it is impossible to setup a USAXS beamline for operation in the SAXS regime and to expect high throughput because of optical mismatch.

${ }^{3}$ The MiniMat ${ }^{\circledR}$ Tensile Tester of Rheometrics Scientific is no longer produced. An Instron ${ }^{\circledR} 4442$ is operated on a regular basis at the polymer beamline X27C of the NSLS in Brookhaven, USA [6]. The small Zwicki (R) (Z1.0/TH1S, Zwick GmbH, Ulm, Germany) is of similar size, and space problems may arise in cramped experimental hutches.
} 
experiments have fostered important developments in the field of SAXS theory. Exemplary, the fundamental work of POROD [10,11], BONART [12-14] and VONK [15] shall be mentioned.

Through all the years quasi-dynamical studies employing the stretch-hold principle [1] represent the predominant fraction of all the investigations on the nanostructure evolution of composite materials $[6,16-35]$. Since this technique means that the sample is held at a constant elongation until a low-noise scattering pattern has been exposed, the data can, in principle, be analyzed by quantitative evaluation, although most of the published papers do not proceed far beyond a presentation and interpretation of the accumulated images.

Genuine dynamical in situ investigations of deformation have been carried out since the 90ies [36] using an instrument [37] at the SRS in Daresbury. Exploiting the shortest available cycle time of $40 \mathrm{~ms}$ with strongly scattering materials, it is possible to record 2D patterns that are sufficient for qualitative interpretation. In this environment structure evolution can be studied at strain rates of $\dot{\varepsilon} \approx 10^{-1} \mathrm{~s}^{-1}$, which are common in industrial processes. A selection of the studies performed with this instrument [2-5] demonstrates both its power and its limits.

To our knowledge the first paper based on high quality data with an exposure of $15 \mathrm{~s}$ and a cycle time of $30 \mathrm{~s}$ has recently been published by CHEN et al. [38].

\section{Scattering theory and materials structure}

Reference to textbooks. In order to establish the relation between the structure of matter and the corresponding X-ray scattering pattern a two-step deduction is carried out (cf. textbooks, e.g. [39-43]).

In the first step the interaction of X-rays with a single electron (in some absorbing matter) is considered, elaborating all the deviations of the practical interaction from the paradigm of a photon that is interacting elastically with a pseudo-electron that, in return, is emitting a spherical wave. As a result, several corrections should be applied to the measured raw data before a quantitative analysis ${ }^{4}$ in order to make them satisfy the paradigm. These corrections comprise absorption correction, polarization correction, correction for Compton scattering, suppression of fluorescence and multiple scattering [44]. In practice, absorption correction should be applied in general. Polarization correction is important for the WAXS, as is a subtraction of the Compton scattering background [41]. A test for multiple scattering [44] is important, if porous materials are studied by SAXS. It is very important in the field of USAXS.

In the second step the arrangement of the electrons (i.e. the structure of matter) is added, and the Fourier relation of the structure to the scattered X-ray intensity $I(\mathbf{s})$ is established by the so-called kinematic scattering theory.

The Fourier transform. According to the Fraunhofer approximation of kinematic scattering theory, the real space and the reciprocal space are related to each other by an integral transform known by the name Fourier transform. It shall be indicated by the operator $\mathscr{F}()$. The $n$-dimensional $(\mathrm{nD})$ Fourier transform of $h(r)$ is defined by

$$
\mathscr{F}_{n}(h)(\mathbf{s}):=\int h(\mathbf{r}) \exp (2 \pi i \mathbf{r s}) d^{n} r,
$$

with $i$ the imaginary unit - and back-transformation simply yields

$$
\mathscr{F}_{-n}(H)(\mathbf{r}):=\int H(\mathbf{s}) \exp (-2 \pi i \mathbf{r s}) d^{n} s,
$$

\footnotetext{
${ }^{4}$ Quantitative analysis means that the shape of the intensity function is considered or entered in a numerical evaluation procedure. For a simple qualitative discussion (peak positions, strong intensity changes) corrections are negligible.
} 
with $H(\mathbf{s}):=\mathscr{F}_{n}(h)(\mathbf{s})$. In the field of scattering 1D-, 2D- and 3D-transforms are required. The kernel of the Fourier transform is called the harmonic function

$$
\exp (2 \pi i r s)=\cos (2 \pi r s)+i \sin (2 \pi r s)
$$

and the Fourier transform is said to perform an harmonic analysis.

In the q-system the pair of transformations

$$
\mathscr{F}_{n}(h)(\mathbf{q}):=\int h(\mathbf{r}) \exp (i \mathbf{r q}) d^{n} r
$$

and

$$
\mathscr{F}_{-n}(H)(\mathbf{r}):=\left(\frac{1}{2 \pi}\right)^{n} \int H(\mathbf{q}) \exp (-i \mathbf{r q}) d^{n} q
$$

is asymmetric with different pre-factors that must be tracked in calculus.

Structure and scattering in a nutshell. The fundamental relations between the electron density distribution inside the sample, $\rho(\mathbf{r})$, and the observed scattering intensity, $I(\mathbf{s})$ are conveniently combined in a sketch

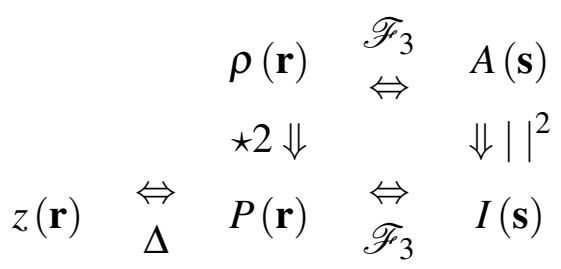

from which the theoretically explored options for a quantitative analysis of the scattering, $I(\mathbf{s})$, can be accessed. According to the scheme, the real-space electron density, $\rho(\mathbf{r})$, by 3D complex Fourier transform is converted into the scattering amplitude, $A(\mathbf{s})$, in reciprocal space.

Stepping downwards in the scheme from the amplitude, we arrive at the scattering intensity

$$
I(\mathbf{s})=|A(\mathbf{s})|^{2}
$$

by taking the square of the absolute value. The unidirectional downward arrow shows that this operation cannot be reversed. We are loosing the phase information of the structure. This means that we cannot reconstruct absolute positions of individual domains (i.e. crystallites) in the material. Only relative distances among domains, i.e. their correlations are readily determined. As a consequence of the last-mentioned operation, the intensity

$$
I(\mathbf{s})=I(-\mathbf{s})
$$

is always an even function (point symmetry) with real (not complex) values. In practice, this means that we can reconstruct $I(\mathbf{s})$ from $I(-\mathbf{s})$ and vice versa, if only one of these points has been measured on the detector. Another consequence is relaxing the calculus, as long as we stay with the s-system: When switching back and forth between reciprocal and real space by means of the Fourier backtransform and the Fourier transform, even the switching sign in the harmonic kernel becomes negligible.

Proceeding to the left along the bottom edge of Eq. (6) we arrive back in real space at the Patterson function,

$$
P(\mathbf{r})=\mathscr{F}_{3}^{-1}(I(\mathbf{s})) \text {. }
$$

The physical meaning of the Patterson function is readily established by introduction and interpretation of the autocorrelation operation $\star 2$

$$
P(\mathbf{r})=\rho^{\star 2}(\mathbf{r})
$$




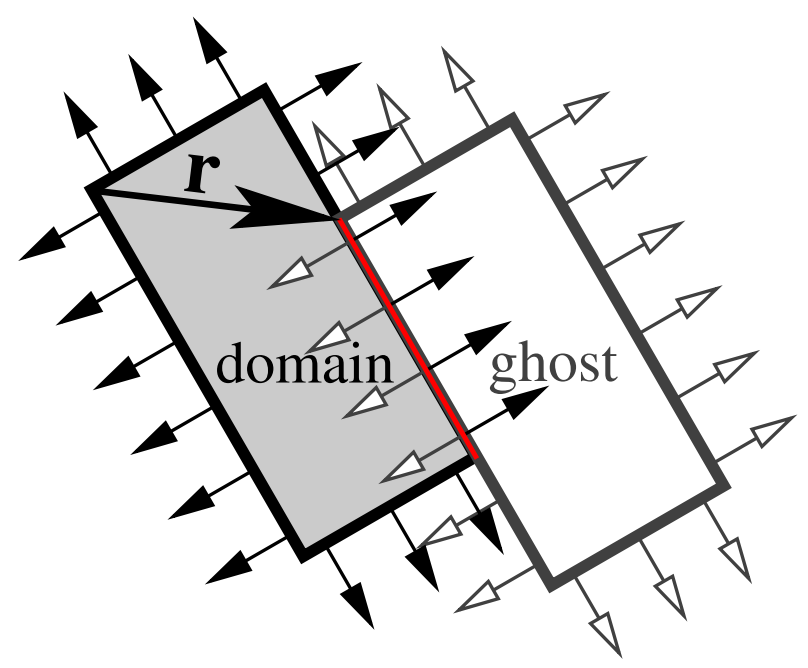

Figure 1. A particle-ghost autocorrelation of gradient vectors is generating the CDF. These vectors are emanating in normal direction from the surfaces of the particle and its displaced ghost. The ghost is displaced by the vector $\mathbf{r}$. The scalar product of the gradient vectors is vanishing everywhere, except for the regions with surface contact between particle and ghost

which turns the structure $\rho(\mathbf{r})$ directly into the Patterson function. Because the autocorrelation integral expands into

$$
\rho^{\star 2}(\mathbf{r})=\int \rho(\mathbf{y}) \rho(\mathbf{r}+\mathbf{y}) d^{3} y,
$$

it is identified by the overlap integral between the structure, $\rho(\mathbf{y})$, and its displaced ghost. Here the vector $\mathbf{r}$ describes the amount and the direction of the displacement. In particular in the field of SAXS a common synonym for the Patterson function is the correlation function (Debye (1949) [11], Porod (1951) [10]),

$$
\gamma(\mathbf{r})=\rho^{\star 2}(\mathbf{r}) / \rho^{\star 2}(0)=P(\mathbf{r}) / \rho^{\star 2}(0) .
$$

By its normalization $\gamma(0)=1$ it indicates that the correlation between structure and ghost is perfect, if there is no displacement at all.

Finally, the structure of soft matter frequently may be considered to be made from domains which can be distinguished from each other by a sufficient difference of their electron densities (contrast). This is the case for materials comprising soft and hard domains, voids, crystallites, or amorphous regions. In this case it has proven advantageous not to study the correlation function, but to perform edge enhancement

$$
z(\mathbf{r})=\Delta P(\mathbf{r})
$$

by applying the Laplacian operator. A method for the computation of a 3D chord distribution function (CDF), $z(\mathbf{r})$, has been described in 2001 [45, 46], but before that a one-dimensional chord distribution called interface distribution function (IDF), $g_{1}(r)$ had already been proposed by Ruland [47-49] for the study of lamellar systems. The basic idea of this approach is even reaching back to 1965, when Méring and Tchoubar [50-53] proposed the (radial) chord length distribution (CLD), $g(r)$.

Comparing the IDF to the CDF, the $1 \mathrm{D}$ derivative $d / d r_{3}$ of the electron density $\rho\left(r_{3}\right)$ is replaced by the gradient $\nabla \rho(\mathbf{r})$, as is the second derivative $d^{2} / d x^{2}$ in the IDF by the Laplacian $\Delta$ in the CDF [45]. In analogy to the particle-ghost construction of the correlation function (cf. textbooks $[43,44,54]$ the construction of the CDF is readily demonstrated (Fig. 1). In a multiphase material the gradient field $\nabla \rho(\mathbf{r})$ is vanishing almost everywhere. Exceptions are 
the domain surfaces. They are continuously populated with gradient vectors, the lengths of which are proportional to the heights of the density jumps. Thus, the autocorrelation among the edge-enhanced gradient field of the structure and its ghost as a function of ghost displacement, $\mathbf{r}$, is computed from the scalar product of the gradient vectors. Approximately it is proportional to the area of surface contact and the product of the density jumps at the contact surface. Its sign is indicating, if there is inner or outer contact. By definition of the CDF, outer contact is carrying the positive sign.

\subsection{Relation Between a CDF and IDFs}

Every radial, 1D slice (cut, section) through the center of a CDF

$$
\lceil z\rceil_{1}\left(r_{\psi, \varphi}\right)=g_{1}\left(r_{\psi, \varphi}\right)
$$

is an IDF, by definition. In the above equation, the slicing direction is indicated by a polar and an azimuthal angle, $\psi$ and $\varphi$, respectively. Of particular practical interest for the study of fibers is the cut of the CDF along the fiber axis,

$$
\lceil z\rceil_{1}\left(r_{3}\right)=z\left(0, r_{3}\right)=g_{1}\left(r_{3}\right)
$$

which describes the longitudinal structure of the material. In analogous manner the transverse structure of the fiber is described by the $2 \mathrm{D}$ slice

$$
\lceil z\rceil_{2}\left(r_{12}\right)=z\left(0, r_{12}\right)=g_{2}\left(r_{12}\right)
$$

of the CDF.

\section{Analysis options derived from scattering theory}

\subsection{Completeness - a preliminary note}

If the structure of an investigated sample can be approximated by a lattice (crystal), the result of the scattering experiment is a diffraction pattern with many distinct peaks - the Fourier transform of an infinite lattice is, again, an infinite lattice of peaks and the success of crystallography is based on the fact that the lattice structure can completely be reconstructed from a limited number of diffraction peaks, if only their positions and strengths are measured.

In general, the structure of soft matter is imperfect and polydisperse. Only in rare cases it can sufficiently be described by a crystal. Thus, in most of the practical cases, an in-depth analysis of scattering intensity by means of the crystallographic approach is not permitted. Although this fact is known for almost a century (Debye and Menke ${ }^{5}$ (1931) [55]), far-reaching conclusions are still drawn, even if they are merely based on determinations of peak positions ("long periods") and peak shapes of scattering patterns.

Soft matter structure is characterized by a continuous density function $\rho(\mathbf{r})$, which is subjected to Fourier transform by the scattering experiment yielding a continuous intensity function $I(\mathbf{s})$ which does not drop to zero in long intervals between narrow peaks. Thus, for any in-depth analysis of distorted structure, either we have to model the complete shape of the pattern, or we have switch to real space, again supplying the respective transform with a complete intensity function.

\footnotetext{
${ }^{5}$ For a translation of the note made by Debye and Menke cf. [44],p. 1
} 


\subsection{Analysis options}

Options of data analysis can be deduced from Eq. (6) and our notions concerning the structure. As an example let us consider the case of small-angle X-ray scattering. In this area the structure is described by a continuous density function. Although there is no ${ }^{6}$ way back from intensity to density, there are several options for data analysis:

1. Utilize theory and find out, how some structure parameters can be determined from the intensity directly,

2. walk from the intensity along the lower edge half-way back to real space, where the transformed data are closer to human perception,

3. model a structure and fit it to the intensity or

4. in addition to item 2 carry out "edge enhancement" in order to visualize structure by means of the chord distribution function $(\mathrm{CDF}), z(\mathbf{r})$, and interpret or fit it.

Effort of data analysis. The above-mentioned options are listed in the order of increasing complexity for the scientist. When scattering curves (isotropic data) shall be analyzed, all the four listed options have proven to be manageable by many scientific groups.

In contrast, a real challenge is the analysis of scattering images from anisotropic materials, and in this subarea many scientists surrender and resort to the interpretation of peak positions and peak widths in raw data.

A shortcut solution for the analysis of anisotropic data is found by mapping scattering images to scattering curves as has been devised by Bonart in 1966 [13]. Founded on Fourier transformation theory he has clarified that information on the structure "in a chosen direction" is not related to an intensity curve sliced from the pattern, but to a projection of the pattern on the direction of interest.

The barrier to the application of the shortcut is probably resulting from the need to pre-process the scattering data and to project the 3D scattering intensity. This task requires 3D geometrical imagination and knowledge of methods of digital image processing, a field that is quite new to the community of scatterers. Programmers, on the other hand, are rarely educated in the fields of scattering and multi-dimensional projections.

\subsection{Parameters, functions and operations}

The scheme of Eq. (6) which is sketching the relation between structure and scattering intensity contains many parameters, functions and operations which require explanation:

$V \quad$ is the irradiated volume. It is defined by the sample thickness multiplied by the footprint of the incident primary beam on the sample.

$\rho(\mathbf{r}) \quad$ the electron density difference

$A(\mathbf{s}) \quad$ scattering amplitude

$k=\rho^{\star 2}(0)$ scattering power

$Q=k / V \quad$ invariant

$P(\mathbf{r}) \quad$ Patterson function

\footnotetext{
${ }^{6}$ Except for the case of anomalous SAXS
} 
$\gamma(\mathbf{r})=P(\mathbf{r}) / k$ SAXS correlation function

$z(\mathbf{r}) \quad$ chord distribution function $(\mathrm{CDF})$

The X-ray detector measures the intensity of electromagnetic waves, i.e., the absolute square ||$^{2}$ of their amplitude. Thus, in combination, the upper path between density and intensity through the square is written as

$$
I(\mathbf{s})=\left|\mathscr{F}_{3}(\rho(\mathbf{r}))\right|^{2} .
$$

In the lower path through the square we have an equivalent formulation

$$
I(\mathbf{s})=\mathscr{F}_{3}\left(\rho^{\star 2}(\mathbf{r})\right)
$$

with the Patterson or correlation function $\rho^{\star 2}(\mathbf{r})$ involved. $\rho^{\star 2}(\mathbf{r})$ is generated from the "inhomogeneities" $\rho(\mathbf{r})$ by means of the autocorrelation.

\section{The experiment}

\subsection{Principal design}

X-ray source: Laboratory vs. synchrotron. Concerning the output power, not only synchrotron beamlines, but also laboratory sources have experienced a tremendous progress in the previous decade. Details have been described in my textbook [44]. Anyway, for a study of nanostructure evolution during continuous mechanical tests, the brilliance of a laboratory source will most probably never grow sufficiently high. As a consequence, only experiments following the stretch-hold technique [1] appear to be feasible in the laboratory, even if rotating anodes, Göbel mirrors and high-efficiency detectors are combined. In general, the results of stretch-hold experiments cannot be assigned to dynamic mechanical tests (cf. Fig. 10).

Basic setup. Modern equipment is most frequently collecting small-angle scattering data from a large angular region at the same time by means of a two-dimensional (2D) X-ray detector $^{7}$. In principle the basic setup resembles the traditional photographic X-ray cameras (pinhole camera, Kiessig camera). A sketch is presented in Fig. 2. Such a setup comprises beam monitoring devices (ionization chamber, PIN-diode) and a detector. If the beam monitoring devices are not recording the primary beam intensity as a function of time, there is no chance to normalize the whole series of scattering patterns with respect to constant flux and a constant irradiated volume. In horizontal direction there is ample space to place the tensile tester in the X-ray beam. Problems may arise from missing space in vertical direction, if the beamline construction is based on an optical bench with its bars running in horizontal direction. A more flexible beamline design is "the dance floor" [44].

\subsection{Engineering solutions}

\subsubsection{Setup for mechanical testing}

The sample in the air gap. Figure 3 shows the mounting of tensile testers in the synchrotron beam. The close-up (Fig. 3b) shows a polypropylene (PP) sample clamped between the cross-heads with fiducial marks applied on it. The tester is able to perform symmetrical

\footnotetext{
${ }^{7}$ In the field of SAXS and USAXS (ultra-small angle X-ray scattering) the detector image is equivalent to a rectangular plane in reciprocal space, which is commonly addressed by either the symbol s or by the symbol $\mathbf{q}=2 \pi \mathbf{s}$.
} 
drawing, so that the spot irradiated by the synchrotron beam is not drifting away too fast. Online control of the spot position is not yet possible. A TV camera is monitoring the sample and the fiducial marks. The position of the X-ray beam is indicated on the TV screen by means of a cross-hair cursor (cf. Fig. 6) which is positioned in advance by means of a scintillation screen mounted between the clamps.

Application of fiducial marks. Using a rubber stamp (Fig. 4) appears to be an appropriate and easy way to apply marks to long samples which are uniaxially extended. If the samples are smaller or shall be subjected to biaxial deformation, varnish can be sprayed on the sample that is partly covered by a woven steel-wire screen. Such screens are commercially available for

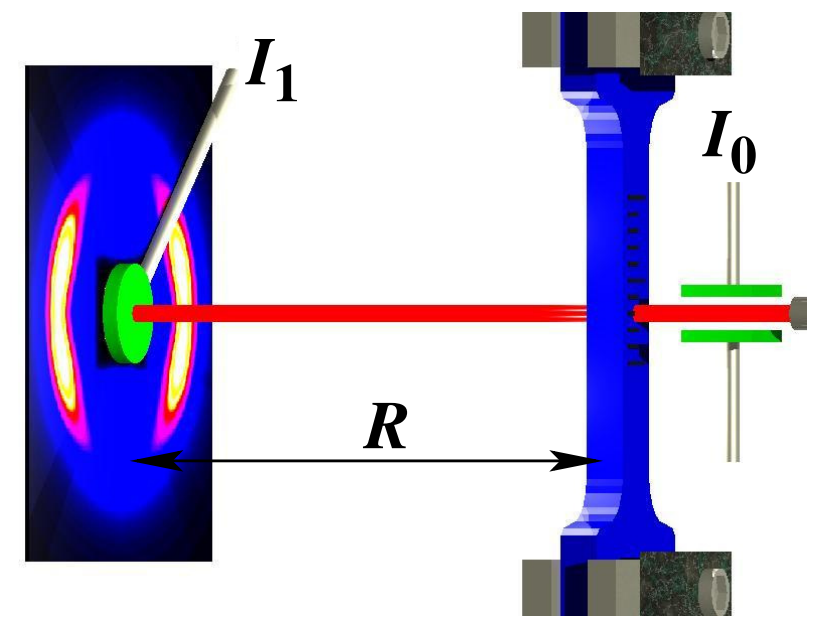

Figure 2. The basic X-ray setup for the study of deformation at a synchrotron. The intensity of the incident X-ray beam is measured in an ionization chamber $\left(I_{0}\right)$. Thereafter it penetrates the sample which is subjected to mechanical load. At a distance $R$ behind the sample the detector is recording the scattering pattern. In its center the detector is protected by a beam stop. It is equipped with a PIN-diode $\left(I_{1}\right)$ which records the intensity of the attenuated beam

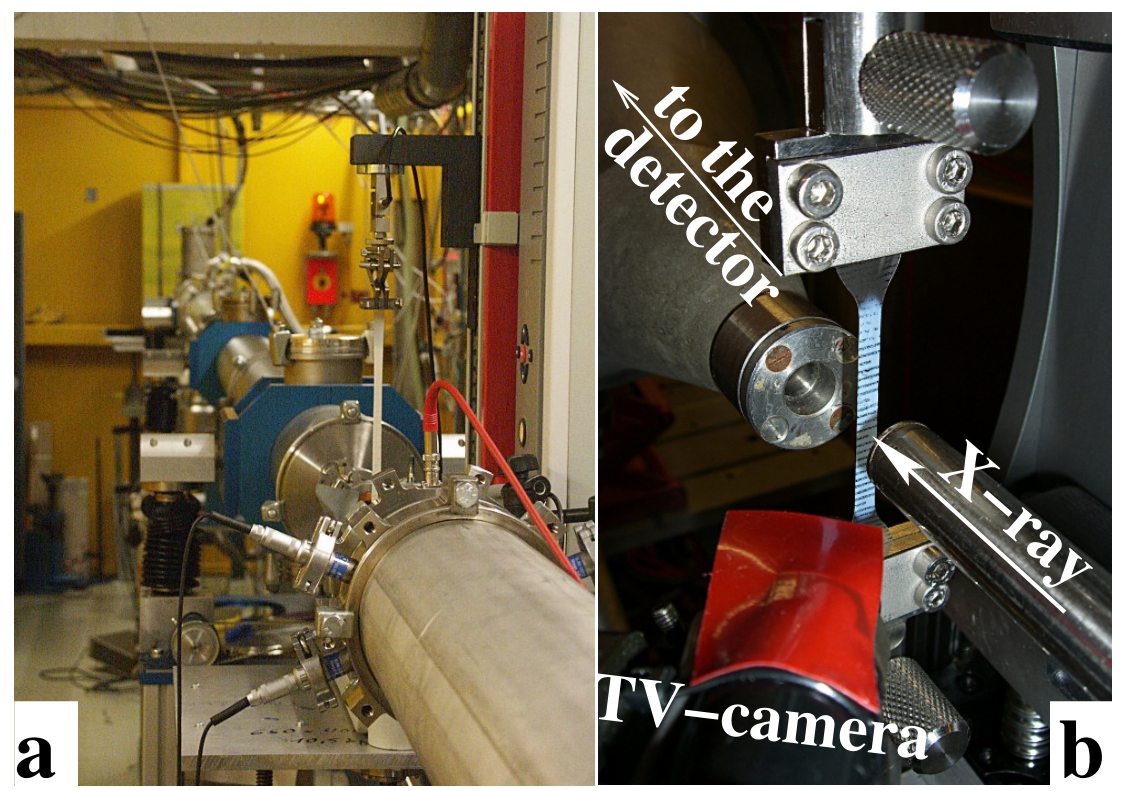

Figure 3. Extensometers at synchrotron beamlines of HASYLAB, Hamburg. (a) Zwicki ${ }^{\circledR}$ Z1.0/TH1S mounted at beamline BW4 (b) Close-up view of a customized extensometer in the X-ray beam of beamline A2. Polypropylene test-bar (DIN 53504) between the cross-bars. TV camera monitoring the sample for the true elongation 
mesh-widths down to $25 \mu \mathrm{m}$ and can easily be cleaned (cf. Fig. 5). Each of these methods as its drawback: The stamp ink will not dry on some materials, whereas dry spray varnish is prone to flaking off the sample upon straining.

During the experiment its progress is observed on monitors (Fig. 6) outside the experimental hutch. One of the monitors shows the TV-image of the sample with the penetration zone of the synchrotron beam indicated. For every SAXS pattern that is recorded, at least one image from the video stream is grabbed and stored together with the elapsed time. Further monitors display the actual SAXS pattern, the progress of the experiment reflected in the engineering stress-strain curve, and the testing program. Time, force and the distance of the cross-heads are continuously written into an ASCII file. From this file the force can directly be associated to each scattering pattern. The true elongation must be determined manually and after the end of

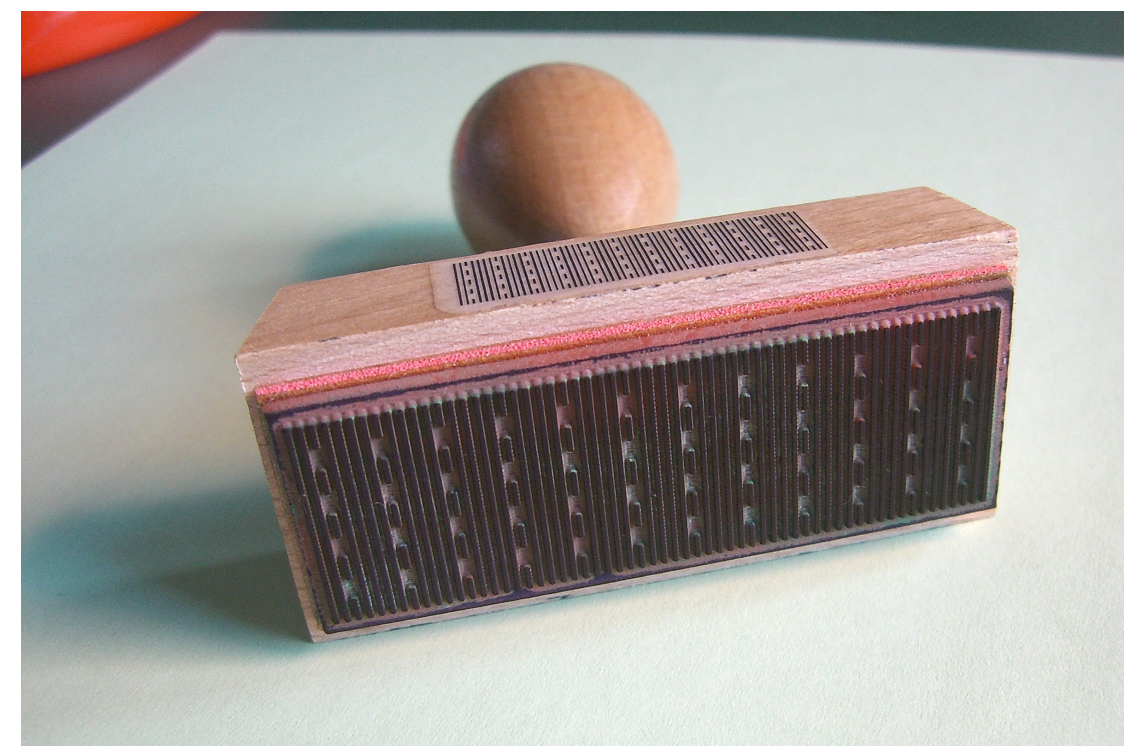

Figure 4. Rubber stamp with a line grid of $2 \mathrm{~mm}$ distance for marking polymer samples to be used in mechanical tests

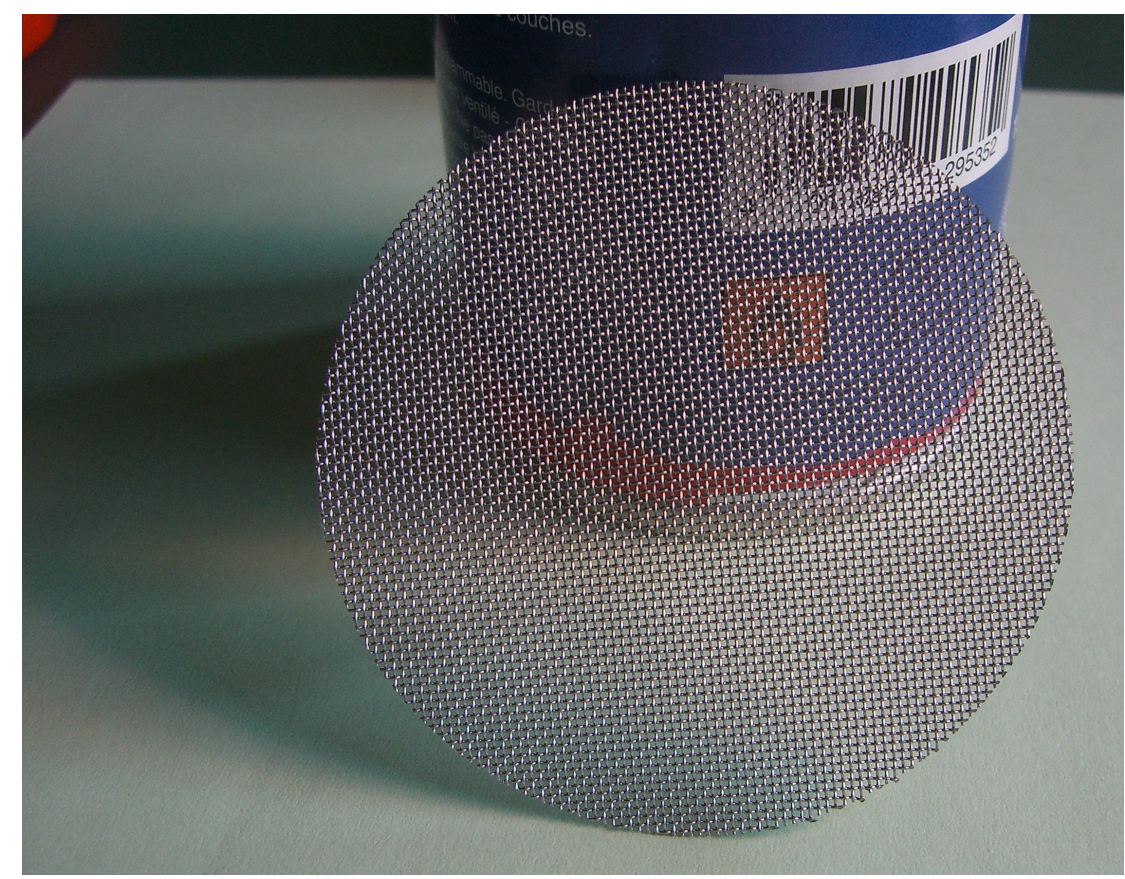

Figure 5. Commercial steel-wire sieve with a mesh width of $0.625 \mathrm{~mm}$ in front of a spray varnish 
the experiment from the distance between the fiducial marks on the images captured from the video.

\subsubsection{Setup for micro-tomography under bending load}

In order to study nanostructure variation as a function of position in the sample, there are synchrotron beamlines with a microbeam, i.e. a X-ray beam with a diameter ranging from $100 \mu \mathrm{m}$ down to hundreds of nanometers. An optical element by which some standard synchrotron beams can be turned into a microbeam is a stack of refracting beryllium lenses [56]. Figure 7 shows such an element mounted in the beam of beamline BW4 at HASYLAB, Hamburg. By means of this installation the ultra-small-angle X-ray scattering (USAXS) beamline with a beam diameter of $1 \mathrm{~mm}$ is turned into a microbeam small-angle X-ray scattering (SAXS) setup. Beam diameters down to $40 \mu \mathrm{m}$ can presently be achieved with this setup.

If a polymer part is translated and rotated in such a microbeam (Fig. 8), the three-dimensional (3D) spatial nanostructure variation in the part can be studied without slicing. For this purpose tomographic reconstruction of the SAXS patterns that would have emerged from tiny volume elements (voxels) in the material is carried out $[57,58]$. Concerning a deformation study, bending load can be applied to a polymer part and the effect to the spatial variation of nanostructure can be studied. The typical beamtime required for the collection of the complete set of projected scattering data is $24 \mathrm{~h}$. A sample bender that has recently been used by us for test experiments is shown in Fig. 9.

\subsection{Scattering data and its evaluation}

Detailed treatment in a text book. Increasingly rarely scattering experiments are carried out by well-trained experts. Thus, there is a high risk of both experiment design flaws and of recording incomplete data. As a result, the analysis of the data is kept cursory or even becomes

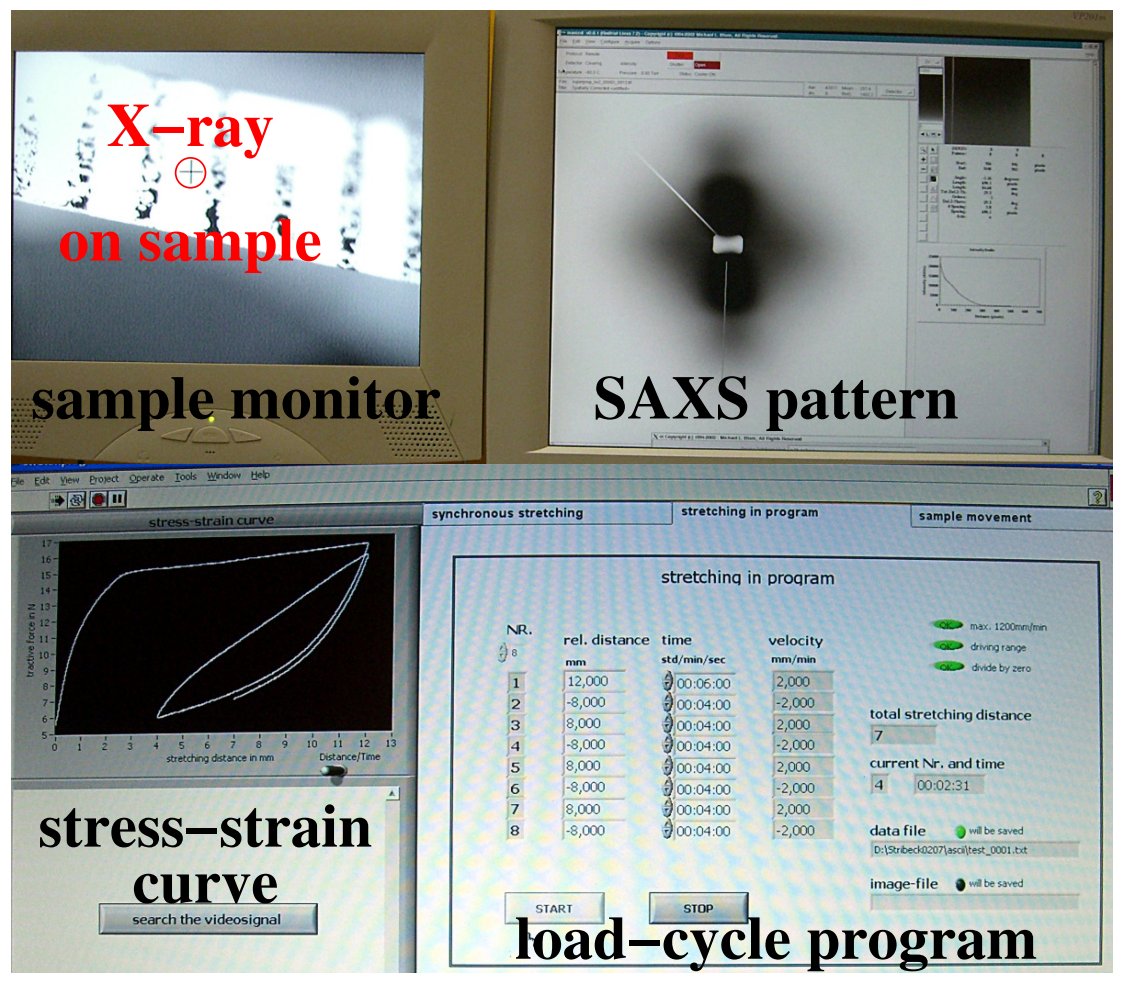

Figure 6. Mechanical testing of polypropylene in progress as watched on monitors at beamline A2, HASYLAB 
erroneous. In my text book [44] the necessary steps concerning interfaces and electronical coupling, the design of the experiment, and the evaluation of the data are described in detail.

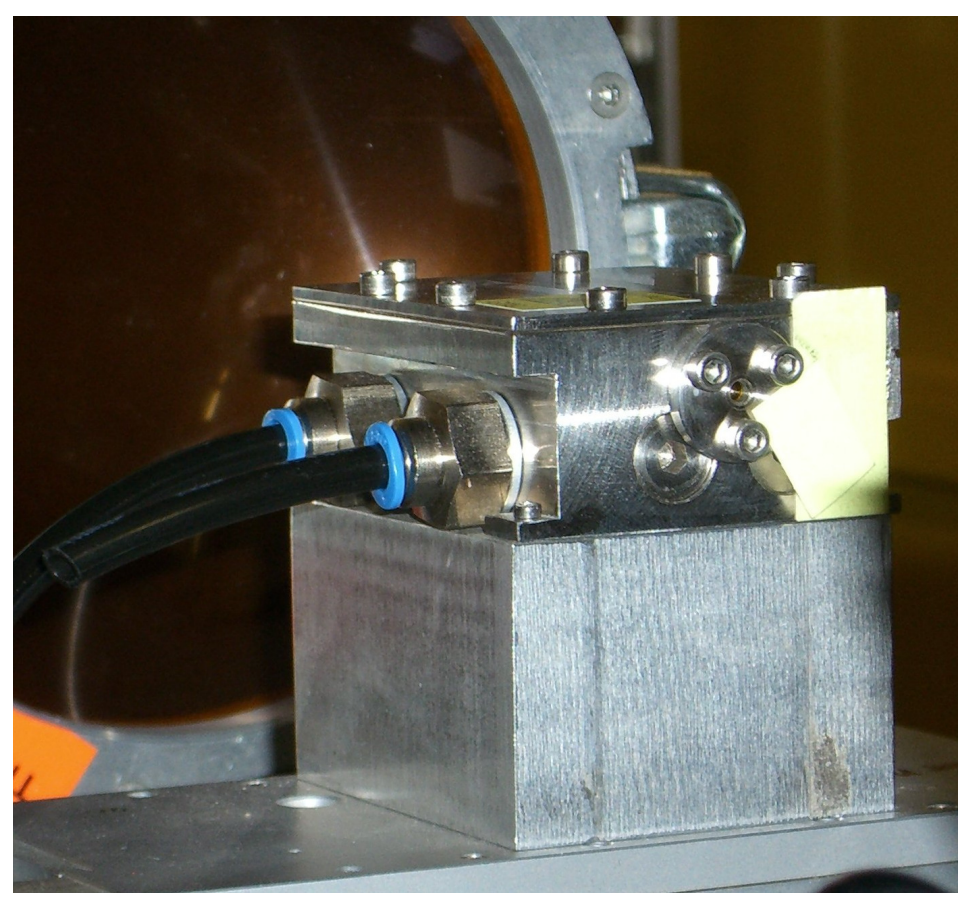

Figure 7. Stacks of refracting Be-lenses are used to demagnify appropriate synchrotron beams. The resulting diameter is in the range of micrometers

(a) side view

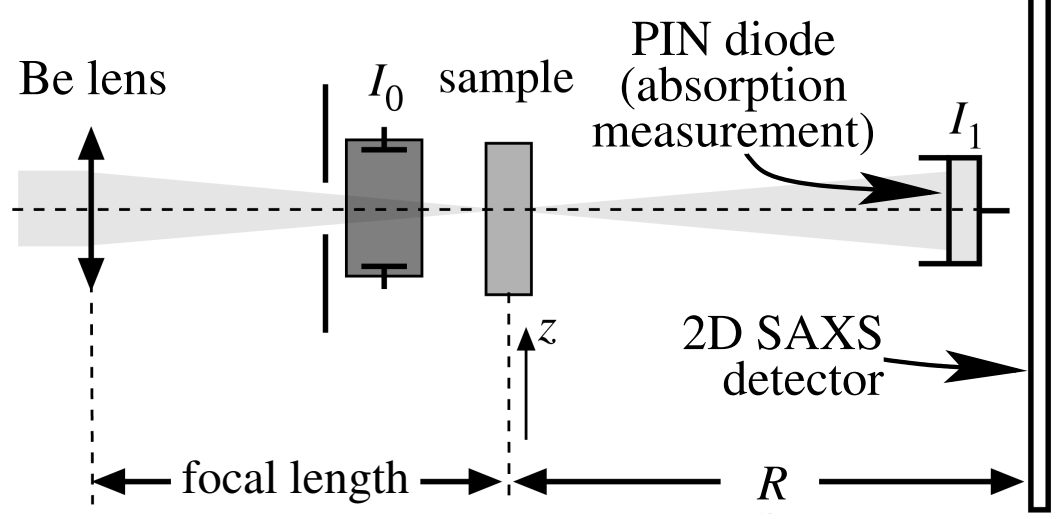

(b) top view of

sample region

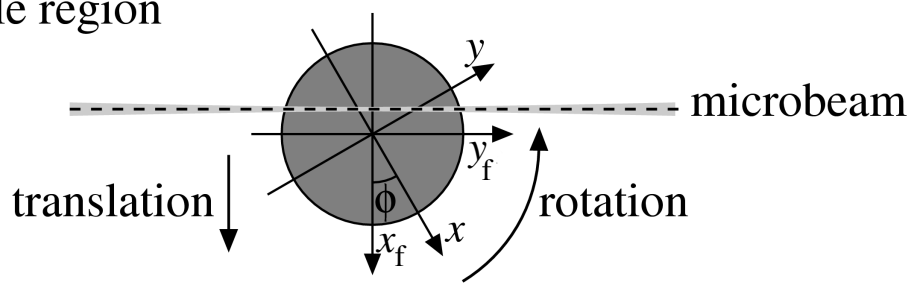

Figure 8. Sketch of a setup for X-ray SAXS micro computer tomography (SAXS $\mu$-CT) and absorption $\mu$-CT. The well-known absorption tomogram is constructed from the signal of the PIN diode, $I_{1} / I_{0}$. The SAXS $\mu$-CT patterns are reconstructed from the projected scattering patterns recorded on the SAXS detector $(R \approx 2 \mathrm{~m})$ while the sample is translated and rotated in the beam 
Here only the important issues concerning proper collection of data are presented.

How to collect complete data sets. After you have collected the first test patterns check that the data files have arrived in the expected directories. Check the size of the files. Open the ASCII files or the ASCII headers in a text editor and check that the environmental data have arrived in the files. Vary environmental parameters in test measurements and check that the values in the ASCII files vary accordingly. If possible, calibrate environmental parameters (e.g., sample temperature, straining force, cross-bar position). Ask the beamline staff to demonstrate what they tell you. Double check! Otherwise your effort may be wasted.

Before the first experiment and after each re-adjustment of the setup the following static data should be collected:

- X-ray wavelength

- sample-to-detector distance $R$

- technical description of the detector(s) used

- size of each pixel cell on the detector

- allocation of monitor channels to environmental parameters

- calibration of monitor channels for which absolute data values are required (e.g., sample temperature, sample elongation)

- a measured primary beam profile or the integral breadths of the primary beam on the detector in horizontal and vertical directions

- in particular in studies of porous or fractal materials, assessment of multiple scattering should be carried out.

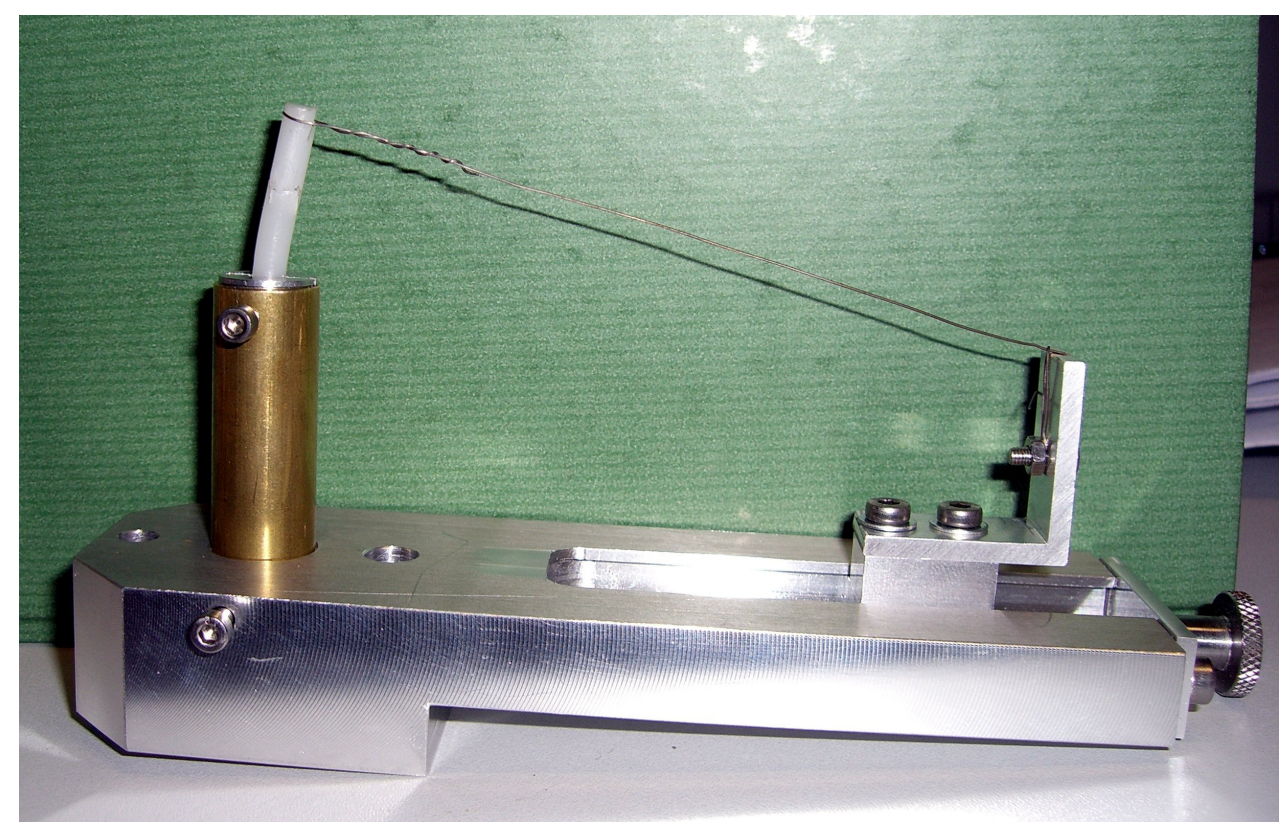

Figure 9. Sample bender for cylindrical polymer rods to be used in conjunction with microbeam SAXS experiments 
The parasitic scattering (machine background) should at least be recorded once within each synchrotron radiation run. You might consider to measure a background before every experiment, because there is some probability that the beam position jumps by a few pixels during refill of the storage ring.

The data evaluation bottleneck. The majority of the users would benefit from a general and user-friendly data evaluation program. Nevertheless, as long as design rules of device interfacing are not respected and the data are not stored in a uniform and simple-to-read data format, this wish will remain a dream. Progress towards a uniform data format is not detectable. On the one hand there is progress in subareas, but on the other hand materials science is fed on experimental innovation the realization of which is introducing new flaws and further complexity.

Flaws in engineering design and setup. Already if one intends to eliminate the machine background from a measured pattern, it is necessary to extract the exposure intervals, primary beam intensities, $I_{0}$, and attenuated intensities, $I_{1}$, from complex data sets which are different at different beamlines. Moreover, the sequence of the stored numbers is changed as a function of the wiring during setup. Thus, if background correction and normalization are not carried out by the beamline staff, some users will not be able to carry out these steps.

On the other hand, if there is a normalization service, the result will be written into a set of files in another proprietary data format, and it may happen that during this process data are not copied which may be essential for further data evaluation (e.g. the elapsed time since start of the experiment, which is the most important parameter in any mechanical test - cf. Fig. 11).

Even more severe flaws are encountered. For example, the student developer of an extensometer control does not hand over the signals from the load cell and from the transducer to channels of the voltage-to-frequency (VDC) converters of the standard beamline electronics, but carries out digitization on hardware of his own with a cycle time of his own. Finally they are stored in an ASCII file on his own computer, using his computer clock as a second time normal. As long as the cycle time is longer than $10 \mathrm{~s}$, a skilled programmer will be able to modify his data pre-evaluation module in such a way that it compensates this flaw.

Curing flaws by computer programming. As the quintessence, the consistent evaluation of streams of scattering data remains to be a computer programming task, and thus, programming skills are required for a successful work with multidimensonal scattering data. Scientists providing these skills will utilize and modify open sources (see for instance [59]). Without these skills even the few popular data evaluation programs like e.g. Fit2D [60] will probably not be employed in an efficient manner.

Automation - the next challenge. In a dynamical experiment hundreds of $2 \mathrm{D}$ scattering patterns are collected which must be evaluated. Thus efficient automation is essential. Instead of writing a thoroughly planned script and running it automatically on all patterns of the series, a user merely trained to operate programs with graphical user interfaces is easily misled. Having carried out the experiment, he will use the computer in accordance to his training: Install a userfriendly evaluation program and start to click buttons without resorting to manual or theory. Operated in such a way, a user-friendly evaluation program turns data-hostile. Moreover, the processing of voluminous data sets turns into an unmanageable task.

For instance, Fit2D is frequently used to extract slices ${ }^{8}$ from $2 \mathrm{D}$ scattering patterns, whereas either an azimuthal average or a projection would have been appropriate. Even such simple and inappropriate operation will turn into a nightmare, when Fit2D is operated by mouse clicks and

${ }^{8}$ Slices are curves that show the intensity along a straight path in the pattern 
many patterns are operated. Finally, noisy slices are extracted instead of low-noise integrated curves. As the consequence, further processing will produce low-significance results.

\section{Techniques: Dynamic vs. stretch-hold}

Only a few years ago dynamic experiments with a good signal-to-noise $(\mathrm{S} / \mathrm{N})$ ratio of the scattering patterns have not been possible. Therefore many of the studies listed in this chapter have been performed by means of the older stretch-hold technique. A recent comparative study [61] unexpectedly revealed pronounced differences after switching from stretch-hold to a slow dynamic experiment. Consequently, only with a lot of cautiousness results on the nanostructure evolution retrieved in stretch-hold studies may be used to explain the dynamic loading process that is relevant in practical applications.

Figure 10 shows in the top row (continuous straining experiment) scattering patterns with narrow and detailed peaks, although the technique implies integration over a considerable interval of the elongation $\varepsilon(\Delta \varepsilon \approx 0.05)$, whereas the scattering patterns accumulated during the same beamtime in the stretch-hold technique at constant elongation appear much more blurred and show a stronger equatorial streak. Thus this comparative experiment demonstrates that during the hold state in a stretch-hold experiment a considerable change of the nanostructure inside the composite may occur.

\section{Advanced goal: Identification of mechanisms}

One of the most important goals of the method is the elucidation of the mechanisms of nanostructure evolution during application of mechanical load. In the previous section it has been demonstrated that for this purpose not the stretch-hold technique, but the recording of scattering patterns during a dynamic materials test should be applied.

Moreover, concerning the mechanical test itself, not only the classical method of continuous straining should be considered, because of the fact that during service a component ${ }^{9}$ made from

${ }^{9}$ Consider, for example, the spare-wheel hutch in a car that shall be made from reinforced polypropylene in order

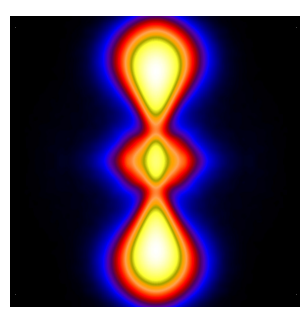

$\varepsilon=0$

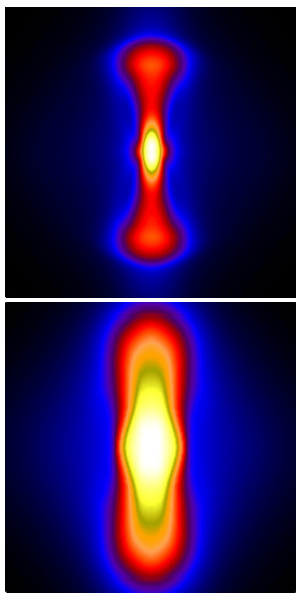

$\varepsilon=0.11$
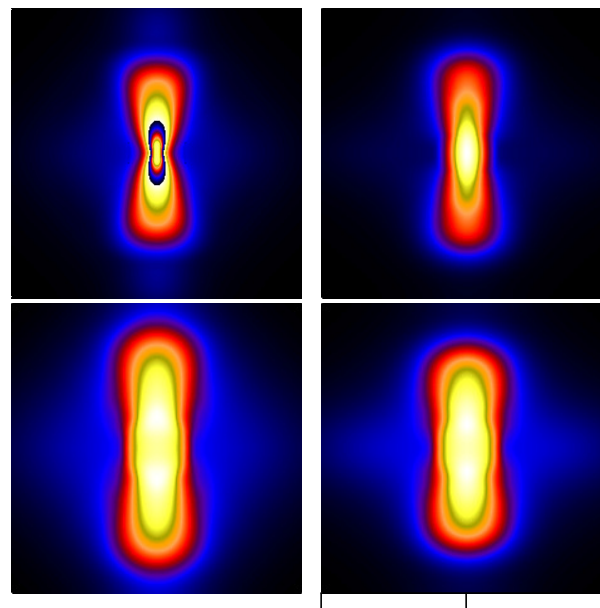

mode of straining: $\dot{\varepsilon} \cong 8 \times 10^{-4} \mathrm{~s}^{-1}$ dynamic

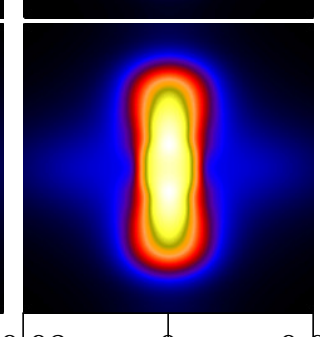

0.08

$s_{3}$ $\left[\mathrm{nm}^{-1}\right]$ $-0$ stretchhold

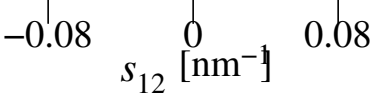

$\varepsilon=0.58$

Figure 10. Comparison between SAXS patterns recorded during continuous straining (top row, $\dot{\varepsilon}=$ $10^{-3} \mathrm{~s}^{-1}$ ) of hard-elastic PP films and results from the corresponding stretch-hold experiment (bottom row) 
a polymer nanocomposite will frequently be subjected to vibrations causing many cycles of alternating load - with materials fatigue becoming the most important reason for failure.

The scientific questions which recently have come into reach shall be demonstrated by results of a recent load-reversal study [62]. Figure 11 displays the macroscopic parameters and some of the nanostructure parameters as a function of the elapsed time. The parameters of the nanostructure have been evaluated from X-ray scattering data.

Experimental. From commercial films of uniaxially oriented, hard-elastic PP tensile bars (DIN 53504) were punched-out and subjected to mechanical load parallel to the direction of the preferential orientation. The thickness of the bars was $50 \mu \mathrm{m}$. Because of the fact that discrete $\mathrm{X}$-ray scattering was observed in a wide angular range, every experiment was carried out both on an USAXS beamline and on a SAXS beamline. USAXS covers the region at extremely small scattering angles and was performed at beamline BW4 of HASYLAB. Measurements in the typical SAXS range were carried out at beamline A2 of HASYLAB. In order to record scattering patterns with good S/N-ratio an exposure of $50 \mathrm{~s}$ was sufficient at both beamlines ${ }^{10}$. Before combination of the pairs of recorded scattering patterns, the SAXS pattern had been deconvolved from its primary beam profile.

to save weight.

${ }^{10}$ After an adjustment of the USAXS beamline for the SAXS range the required exposure increased to $20 \mathrm{~min}$ which is unacceptable. This shortcoming with respect to the asserted universality is probably a problem of the design of the beamline optics. After inserting Be-lenses the SAXS performance of the USAXS beamline is much better.

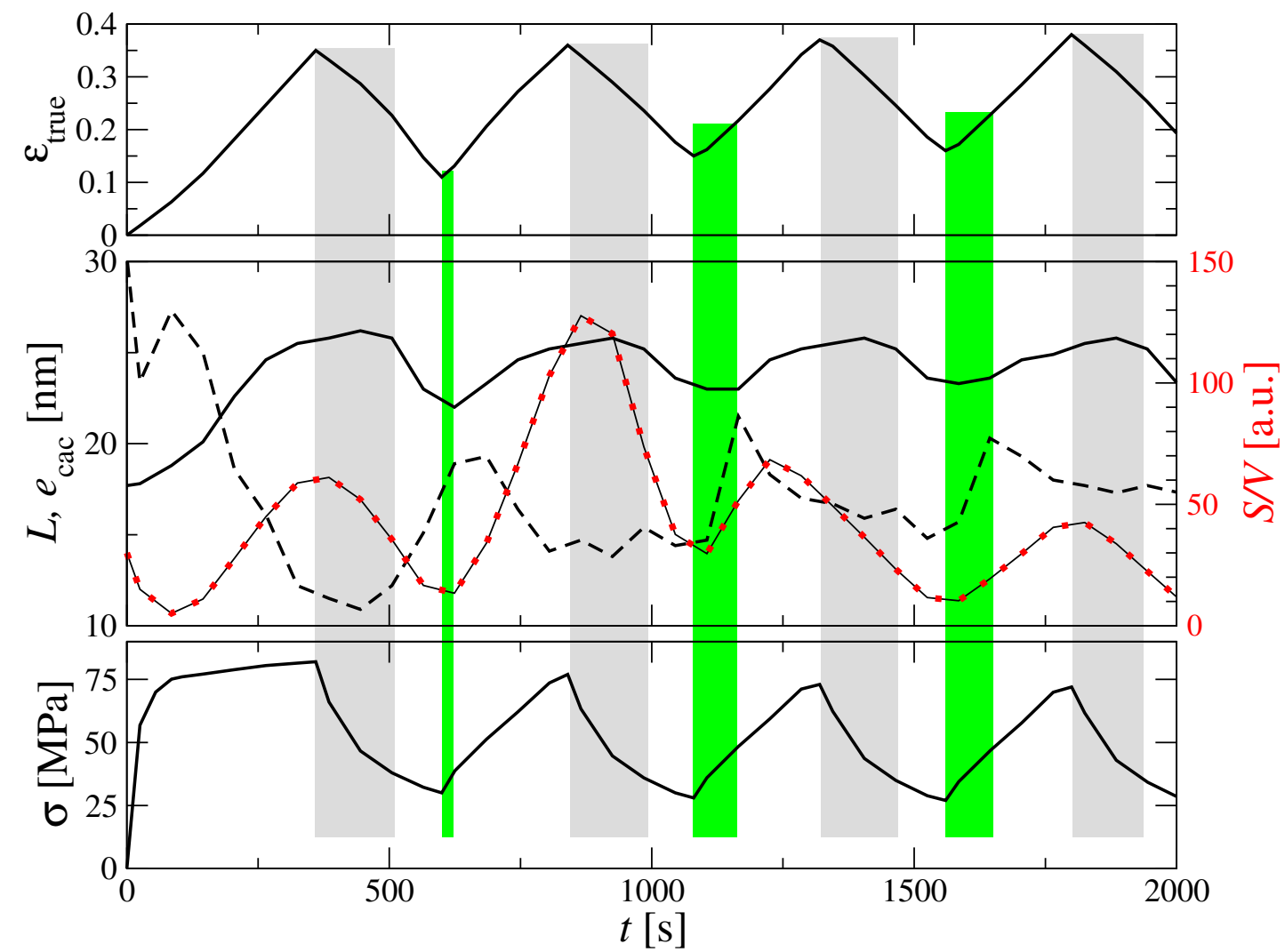

Figure 11. Dynamic load-reversal mechanical test of a hard-elastic PP film. As a function of the elapsed time $t$ nanostructure parameters (diagram in the middle) and the macroscopic parameters elongation, $\varepsilon$ (top), and tensile stress, $\sigma$ (bottom) are displayed. Vertical bars indicate zones of strain-induced crystallization (dark green) and relaxation-induced melting (light gray), respectively 
Data analysis. From the scattering patterns the multidimensional chord distribution function $(\mathrm{CDF})[44,45]$ was computed in order to extract the topological information from the SAXS data. Thereafter the CDF was evaluated, and the curves in the central diagram of Fig. 11 were obtained. The top diagram of Fig. 11 shows the true elongation, $\varepsilon$, measured at the irradiation spot, as induced to the material by the extensometer. The bottom diagram shows the stress, $\varepsilon$, as measured by means of the load cell normalized to the actual cross section of the material.

Results. In the central diagram the solid line presents the long period, $L$, i.e. the average distance between neighbored crystalline lamellae. Obviously, this distance remains constant for a while when relaxation sets in (gray bars). In the first straining stage the long period is increasing monotonously with increasing $\varepsilon$. This finding is unspectacular, as macroscopic strain is almost proportional to nanoscopic strain. The behavior is changing during the subsequent straining stages, as at their beginning a plateau is formed, which is becoming broader with every cycle (dark vertical bars).

If the scattering patterns would have been interpreted directly and the CDF analysis would not have been performed, no further information on the nanostructure would have been obtained and we would have to leave it at the description of a strange phenomenon. Fortunately, from the CDF additional data on the nanostructure can be extracted with little effort. $e_{c a c}$ is the lateral extension of a sandwich made from two crystalline lamellae with an amorphous zone in between ${ }^{11}$. This quantity is a measure of the lateral extension of the average lamella and is reported by a dashed line in the diagram. It is observed that $e_{c a c}$ is decreasing considerably during the first straining phase - lamellae are fragmented into narrow blocks. Its extension remains almost constant during all the relaxation phases. However, in the beginning of each of the following straining phases $e_{c a c}$ is increasing in a sudden burst, an effect that is readily explained by the fast lateral lamellae growth which is known from polymer crystallization. Upon further straining the lateral extension of the crystallites is, again, decreasing. Even with this second nanostructure parameter there would be ample room for speculation on the fundamental mechanism by which the material reacts upon strain.

Before we start the interpretation, let us consider the total integral of the CDF, which, to a first approximation, is proportional to the total surface $S$ of the crystalline domains with respect to the volume $V$ irradiated by the $\mathrm{X}$-ray beam (curve marked with circular symbols). Now we see, that in the beginning of the experiment crystallites are "mechanically destructed" up to the point where the stress-strain curve reaches the yield plateau. From this point on straininduced crystallization sets in and the number of crystals is strongly increasing. As the material is subjected to relaxation, the number of crystals is falling off, again.

Interpretation. From the combination of these results conclusions can be drawn. Only until the material enters the yield-plateau it can be considered a nanocomposite solid body, the crystalline component of which is destroyed by the applied strain. Beyond this point a description of the nanostructure in terms of mechanical categories appears to be inadequate, since the main mechanism by which the material reacts upon mechanical load is strain-induced crystallization and relaxation-induced melting. Moreover, the mechanism of fatigue is already indicated to some extent in the presented data: Concerning the extracted nanostructure parameters, the amplitude of their oscillations is decreasing from cycle to cycle. Thus, the material "is learning" a nanostructure with minimum resistance to load cycling, and favorable materials properties are continuously decreasing.

\footnotetext{
${ }^{11}$ Granted - in principle it is possible to determine the lateral extension of the single lamella, as well. Concerning the PP studied here the determination is difficult, because the corresponding peak is interfering both with the scattering effect of crystalline blocks and that of cross-hatched lamellae, but in this example we do not commit ourselves to the description of all the aspects of the complex nanostructure evolution of the studied material.
} 
Outlook. At this point of understanding and with the dynamic X-ray method at hand, it appears promising to stabilize a favorable nanostructure of the polymer by fitting in a nanocomponent which is not melting and turns polypropylene into a low-fatigue material.

\section{Observed promising effects from stretch-hold experiments}

Even though the older stretch-hold experiments can hardly be used for a reliable description of nanostructure evolution under mechanical load, the observed general scattering effects indicate, in which subareas of the structure analysis of nanocomposites there is a good chance for successful application of the X-ray method. Thus, some examples are presented.

\subsection{Orientation of nanofibrils in highly oriented polymer blends by means of USAXS}

The reinforcement of a fiber material with uniaxially microfibrils (a composite material) oriented parallel to the fiber axis suffers from a serious disadvantage, namely the extremely high anisotropy of the mechanical properties. This situation can be overcome by inclining the reinforcing microfibrils with respect to the principal axis of the material. This principle is observed in the nature where reinforcing fibrils are frequently inclined with respect to the principal axis of fibers found both in fauna [63] and flora [64,65].

Man-made model systems that both show similar scattering patterns, and in which the microfibrillar angle can easily be adjusted have been prepared and a feasibility study [66] has been performed utilizing USAXS at HASYLAB, beamline BW4 in different states of elongation. The studied materials are bundles of about 1000 highly oriented filaments with each a diameter of $30 \mu \mathrm{m}$. The initial microfibrillar angle is adjusted by twisting the bundle more or less. Each filament is a blend of polypropylene/poly(ethylene terephthalate) (PP/PET) (80/20 by wt.) containing a huge amount of PET nanofibrils with diameters of only $60 \mathrm{~nm}$ to $100 \mathrm{~nm}$ and a length of many thousands of nanometers, as documented by scanning electron microscopy. These nanofibrils are homogeneously dispersed in the dominating PP matrix. It is worth to be noted that the studied bundles of filaments may easily be welded together to form a compact fiber without losing their unique orientation. This welding is achieved by annealing the bundles at a temperature between the melting points of PP and PET. Figure 12 presents some of the USAXS patterns. The left column displays the materials before the application of strain. The pattern of the twisted sample is shown in the bottom. The double-tilted equatorial streak due to the twisting of the filaments is clearly visible. The preferential microfibrillar angle $\chi=4^{\circ}[64,65]$ is easily extracted from the inclination of the streak with respect to the equator of the bundle.

The middle column shows the patterns of both samples at an elongation of $20 \%$. While the pattern of the straight-bundle sample appears virtually unaffected, the microfibrils in the twisted-bundle sample are straightened to $\chi=1^{\circ}$ under the load.

The right column displays the USAXS patterns of the two materials after relaxation $\left(\chi=3^{\circ}\right)$. Obviously in the twisted-bundle sample the microfibrillar angle has recovered to a considerable extent. It is interesting to note that the overall elastic recovery of the material with twisted bundles is better than that of the straight-bundle material.

\subsection{USAXS Studies on undrawn and highly drawn PP/PET blends}

In an experiment performed at beamline BW4, HASYLAB [67], highly oriented fiber materials made from blends of the polymers poly(ethylene terephthalate) (PET) and poly(propylene) 

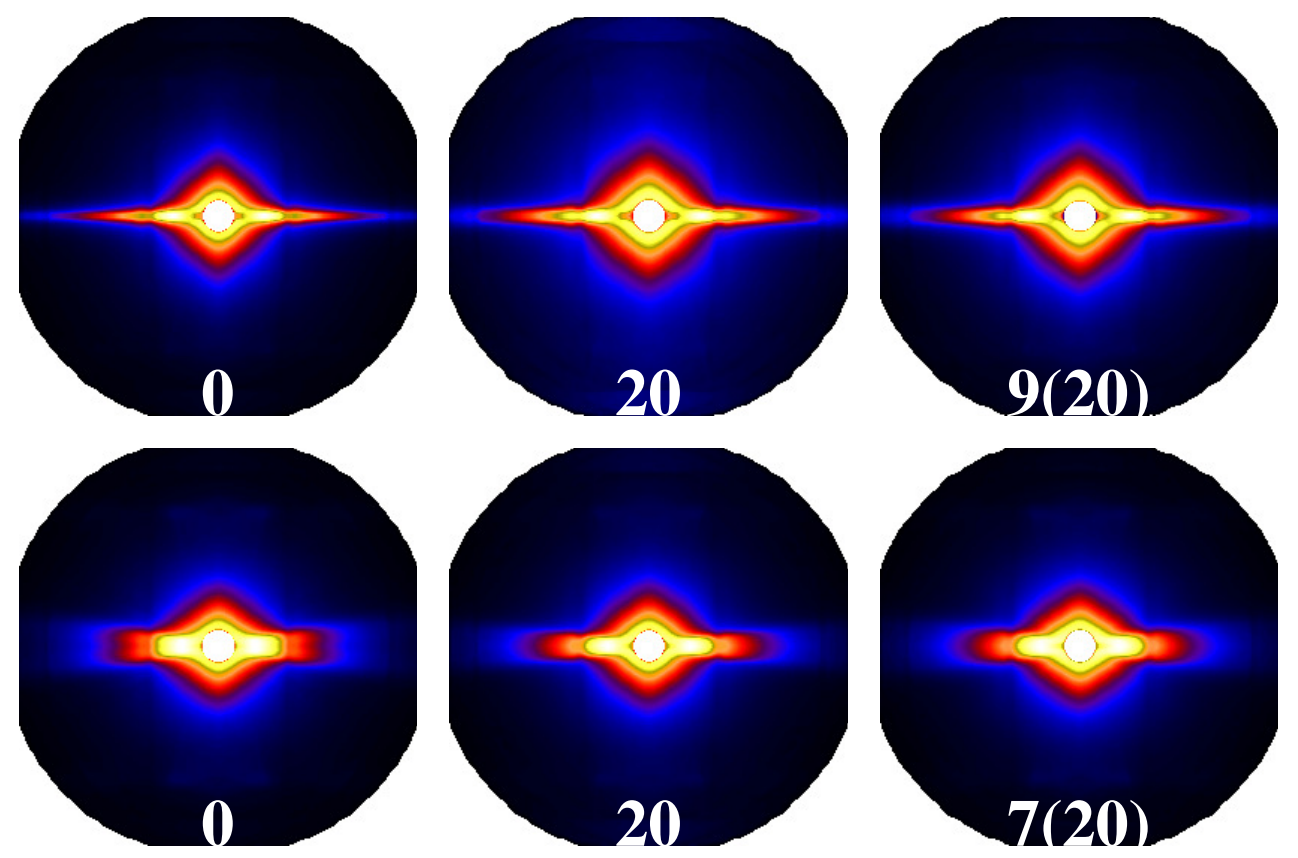

Figure 12. Nanofibrillar PET structure in USAXS patterns (pseudo-color, logarithmic scale) from bundles of fibers made from PP/PET blends by micro-die spinning at different states of elongation. Top row: Untwisted bundle. Bottom row: Twisted bundle. The elongation $\varepsilon$ of the material in percent at which the pattern is taken is indicated in the bottom of each pattern. Right column: For the relaxed material the previous elongation is indicated in parentheses. Fiber axis $s_{3}$ is vertical. Each pattern shows a range $-0.0625 \mathrm{~nm}^{-1}<s_{12}, s_{3}<0.0625 \mathrm{~nm}^{-1}$

(PP) are studied. The materials are prepared in special processes aiming at dispersion of the two components on a nanometer scale. The initial aim has been to study the materials in situ during straining experiments. Because of poor conditions found at BW4 at that time only static measurements of the materials at fixed elongations have been performed. Nevertheless, some conclusions on the materials properties as a function of preparation and elongational load can be drawn.

Dealing with polymer blends, the degree of dispersity is of prime importance because it determines to a great extent the final materials properties. Particularly, the present scientific focus on manufacturing of nanocomposites requires good knowledge of the real sizes of the dispersed particles. The study of domain sizes in the range of a couple of ten nanometers presumes the use of appropriate technique as for example the USAXS.

The samples are neat PP and its blend with PET in a composition PP/PET 80/20 by weight. Fibers are prepared by (1) conventional spinning followed by drawing at $130^{\circ} \mathrm{C}$, and (2) by spinning from a $300 \mu \mathrm{m}$ die without additional drawing resulting in filaments of $30 \mu \mathrm{m}$ diameter. In the latter case bundles of about 300 filaments are irradiated in the experiment.

For all the materials scattering patterns are taken at various deformations in the range between $\varepsilon=0$ and $\varepsilon=0.12$. After relaxation from the deformational steps scattering patterns are taken as well.

In Fig. 13 (left column) some of the USAXS patterns from the neat PP filaments are shown, whereas the middle and the right columns show scattering patterns of the PP/PET blend, respectively.

The scattering patterns from the neat PP (Fig. 13, left column) show diffuse scattering. In addition, the drawn and the elongated material show a faint indication of void scattering (equatorial streak). This is typical for semicrystalline highly oriented homopolymers. The long period reflection $(L=17.5 \mathrm{~nm})$ is observed on the meridian just inside the accessible angular range of 


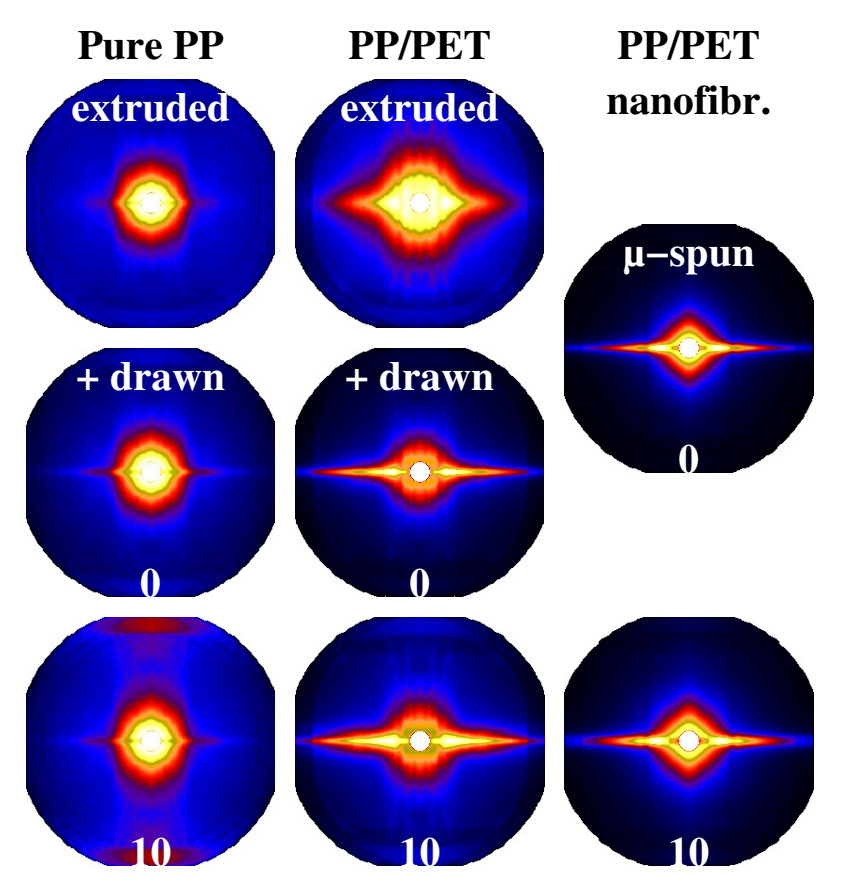

Figure 13. Nanofibrillar PET structure in USAXS patterns (pseudo-color, logarithmic scale) from fibers made from PP/PET blends after different steps of processing. The elongation $\varepsilon$ of the material in percent at which the pattern is taken is indicated in the bottom. Fiber axis $s_{3}$ is vertical. Each pattern shows a range $-0.0625 \mathrm{~nm}^{-1}<s_{12}, s_{3}<0.0625 \mathrm{~nm}^{-1}$

the USAXS. In the middle and the right column the PP/PET blend is shown. Faint long periods are observed in the middle column as well, but not in the right column where the corresponding reflection is outside the USAXS range (i.e. $L<16 \mathrm{~nm}$ ). After conventional extrusion (middle) the fine dispersion of the PET in the PP phase on a nanometer scale is obvious from the size and shape of the central scattering. Drawing (central image) or micro-spinning (right column) leads to the formation of highly elongated needle-shaped PET domains in the PP fiber. Upon drawing of some of the PET nanofibrillar domains appear to break (broadening of the equatorial streaks). The lengths of the nanofibrillar PET domains are much higher, if the material is made by micro-die spinning (right column).

\section{Choosing experiments}

In case the experimental data shall be subjected to quantitative evaluation, the design of the experiment should carefully be chosen. In general, oriented materials with fiber symmetry should be preferred and the fiber axis should run parallel to one of the detector edges (cf. Fig. 2) - otherwise the measured SAXS pattern is incomplete from the point of view of theroy. Nanocomposites which are studied at ambient temperature frequently exhibit both discrete USAXS and discrete SAXS. In this case the mechanical test should be run both at a USAXS beamline, and at a SAXS beamline. Before combining the pairs of patterns, the SAXS pattern must be deconvolved [44, 68-70] from its primary beam profile (cf. Fig. 14). At increased temperature or if the matrix material is polyethylene (PE), a single USAXS experiment may suffice.

\subsection{Experiments with a macrobeam}

Common synchrotron sources offer a beam with a breadth of approx. $2 \mathrm{~mm}$ (Fig. 14). Since this is no microbeam, it shall be called "macrobeam". As shown in the figure, the beam shape is frequently elongated in horizontal direction. Bearing in mind quantitative data analysis, a 


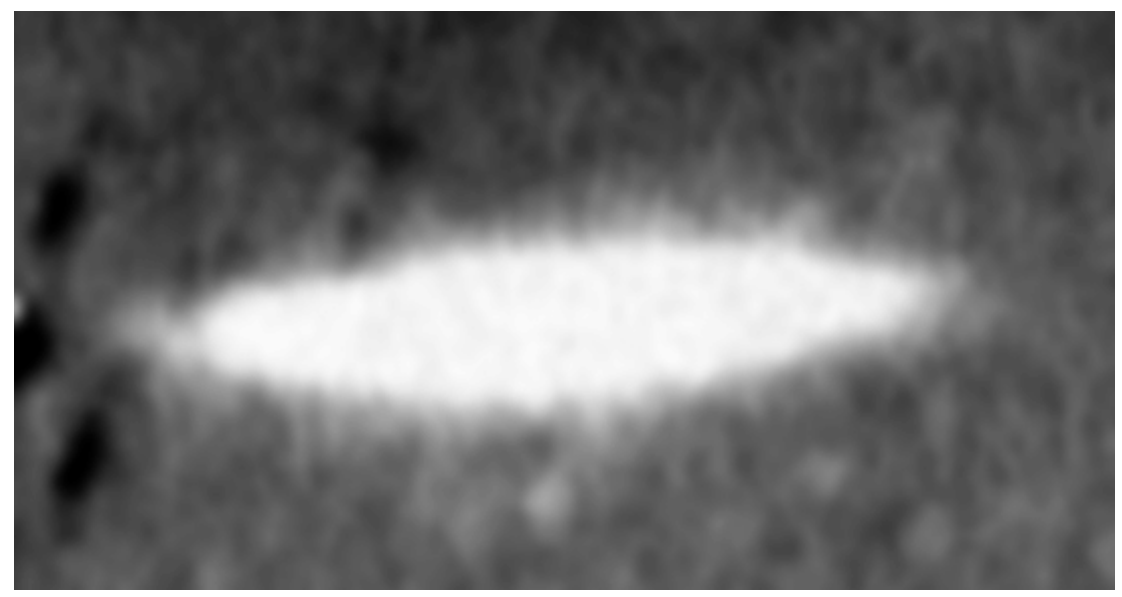

Figure 14. Primary beam profile of beamline A2, HASYLAB, Hamburg recorded on a scintillation screen after optical setup and before mounting of the beam stop and the detector. The horizontal extension of the bright spot is $3 \mathrm{~mm}$

normalization of the scattering patterns can be eased, if the shape and orientation of the primary beam is suitably combined with the shape and orientation of the sample to be studied.

The usual normalization [44] involves several steps. First, the measured machine background is attenuated by the absorption factor. Second, this pattern is subtracted from the measured raw data of the sample. Third, the resulting pattern is normalized for constant $V . V$ is the volume of the sample that is irradiated by the beam, and here the geometrical considerations step in. In mechanical tests, in particular, the irradiated volume is changing due to thinning and thickening of the sample - and the corresponding intensity changes must be compensated so that intensity changes resulting from a geometrical effect are not mistaken for crystallization or melting of domains.

Favorable conditions are present, if either the sample is flat and stays broader than the X-ray beam, or if the material is a fiber with a circular cross section which is crossed in transverse direction by an oblong X-ray beam. The first mentioned case can easily be realized, if the sample is a tensile bare of sufficient breadth. In this case only the sample thickness is changing as a function of load. In the second case, to a first approximation, the intersection between fiber and beam profile yields a truncated cylinder $V$, and the cylinder diameter is varying upon mechanical loading of the fiber.

The variation of the sample geometry as a function of mechanical load is suitably measured in a separate experiment without X-ray beam and verified in the actual X-ray experiment utilizing the variation of the absorption factor $I_{1}(t) / I_{0}(t)$ which can be computed from the data automatically recorded at every beamline which has been set up properly [44].

\subsection{Experiments with a microbeam}

Overview. Microbeam experiments are carried out if the structure in a small volume element of the sample shall be studied. Compared to macrobeam applications the advantage of this method is the possibility to study spatial variations of nanostructure. An example for a matching scientific question is the analysis of the core-shell structure of polymer fibers, or the study of nanostructure variations in nanostructured gradient materials which are developed for medical applications. All the corresponding experiments are carried out in a "scanning microbeam setup".

Of course, there are as well scientific questions which may be answered by time-resolved dynamic experiments with a microbeam. As an example, a dynamical study of the necking 
process could be performed and compared to the corresponding static experiment carried out in a scanning microbeam setup. In the dynamic experiment one would start probing a volume element outside the neck and monitor the passing of the necking front. To-date it should even be possible to vary the strain rate $\dot{\varepsilon}$ to some extent.

2D scanning of thin and flat material. Most of the scanning microbeam studies in which nanostructured material is scanned in 2 dimensions are not investigating material of homogeneous thickness but fibers. Thus, the superposition ${ }^{12}$ of scattering patterns is neglected which, in fact, are originating from regions which are different both with respect of orientation and of nanostructure. A comprehensive survey of the possibilities of the method has been published RIEKEL et al. [71]. A paper by WANG and CAKMAK [72] demonstrates the method in an investigation of microtomed slices of nanocomposites based on PVDF.

SAXS microtomography. The setup for a SAXS $\mu$-CT experiment has been sketched in Fig. 8. Whereas most of the $2 \mathrm{D}$ scanning microbeam papers neglect the variation of nanostructure along the beam path, the $\mu$-CT method makes proper use of the fact that the scattering pattern obtained when irradiating an inhomogeneous sample is a mathematical projection which should not be interpreted directly. Utilizing the well-known method of computer-aided backprojection [57] the scattering patterns originating from an almost homogeneous section (a voxel inside the material) are reconstructed. After that the nanostructure gradient inside the material can be studied [58]. The spatial resolution of the method is limited by the size of the microbeam.

For a practical application one should choose samples with macroscopic fiber symmetry. The axis of sample rotation should coincide with the fiber axis. If the sample is rotated about an axis different from the fiber axis, an effective fiber symmetry will be enforced by rotational averaging (smearing) - in analogy to the rotating crystal method [73,74] from the field of crystallography.

In the near future a broad application of the method is unlikely, because the beamtime required for the recording of a complete set of projections is approximately one day for a single cross section of a sample.

Fibers and reduced microtomography. In a discussion with K. Schneider (IPF, Dresden) we have developed the concept for a "Reduced SAXS $\mu$-CT" method which saves a lot of time: If filaments of cylindrical shape shall be studied, the observed projected scattering pattern is not a function of a rotation angle of the fiber, and the step-wise rotation of the fiber can be saved. Only one translation of the fiber through the microbeam is performed within short time. This is identical to the procedure carried out in the frequently published scanning microbeam studies of fibers. In contrast to the published studies, one would thoroughly choose the step-width for the translational step so that after the experiment the set of scattering patterns would be canalized through the backprojection algorithm in order to retrieve the true nanostructure gradient along the fiber diameter. In order to test the validity of the assumption of fiber symmetry, one could repeat the experiment with the fiber rotated by $90^{\circ}$.

\section{Conclusion and outlook}

Even if in situ investigations of nanocomposites by means of synchrotron radiation and the evaluation of the accumulated stream of scattering data are still a great challenge, the progress during the past decade has shown that now the questions of practical interest can be tackled with

\footnotetext{
${ }^{12}$ In terms of mathematics this superposition is a projection of scattering patterns - and the fact that a complete set of projections can be turned into a complete set of slices (Fourier slice theorem) [44] is the basis of tomographic reconstruction.
} 
a perspective of resilient results. The development of detectors that had remained static for long has quickened considerably after its commercialization. Device developments are advanced by research groups world-wide. Obstacles for a successful exploitation of the methods by a broad community of users are missing standards concerning hard- and software interfaces as well as the shortage of scientists with good programming skills.

\section{References}

[1] Wu J, Schultz J M, Yeh F, Hsiao B S and Chu B (2000) In-Situ Simultaneous Synchrotron Small- and Wide-Angle X-ray Scattering Measurement of Poly(vinylidene fluoride) Fibers under Deformation, Macromolecules 33:1765-1777.

[2] Butler M F, Donald A M, Bras W, Mant G R, Derbyshire G E and Ryan A J (1995) A Real-Time Simultaneous Small- and Wide-Angle X-ray Scattering Study of In-Situ Deformation of Isotropic Polyethylene, Macromolecules 28:6383-6393.

[3] Butler M F, Donald A M and Ryan A J (1997) Time resolved simultaneous smalland wide-angle X-ray scattering during polyethylene deformation: 1 . Cold drawing of ethylene- $\alpha$-olefin copolymers, Polymer 38:5521-5538.

[4] Hughes D J, Mahendrasingam A, Oatway W B, Heeley E L, Martin C and Fuller W (1997) A simultaneous SAXS/WAXS and stress-strain study of polyethylene deformation at high strain rates, Polymer 38:6427-6430.

[5] Blundell D J, Mahendrasingam A, McKerron D, Turner A, Rule R, Oldman R J and Fuller W (1994) Orientation changes during the cold drawing and subsequent annealing of PEEK, Polymer 35:3875-3882.

[6] Liu L Z, Hsiao B S, Fu B X, Ran S, Toki S, Chu B, Tsou A H and Agarwa P K (2003) Structure Changes during Uniaxial Deformation of Ethylene-Based Semicrystalline EthylenePropylene Copolymer. 1. SAXS Study, Macromolecules 36:1929-1929.

[7] Kratky O (1933) On the deformation mechanism of fiber materials. Part I (Ger.), Kolloid Z 64:213-222.

[8] Kratky O (1933) On the deformation mechanism of fiber materials. Part II (Ger.), Kolloid $Z$ 68:347-350.

[9] Kratky O (1938) The computation of micellae dimensions of fiber materials from the interferences diffracted at extremely small angles, Naturwiss 26:94.

[10] Porod G (1951) The Small-Angle X-Ray Scattering from densely packed colloidal systems (Ger.), Colloid Polym Sci 124:83-114.

[11] Debye P and Bueche A M (1949) Scattering by an Inhomogeneous Solid, J Appl Phys 20:518-525.

[12] Bonart R and Hosemann R (1962) Fibrillar Structures in cold-drawn linear polyethylene (German), Kolloid Z u Z Polymere 186:16-29.

[13] Bonart R (1966) Colloidal structures in strained polymers, Kolloid Z u Z Polymere 211:1433.

[14] Bonart R, Hosemann R and McCullough R L (1963) The Influence of Particle Size and Distortions upon the X-ray Diffraction Patterns of Polymers, Polymer 4:199-211.

[15] Vonk C G (1979) A SAXS study of PE fibers, using the two-dimensional correlation function, Colloid Polym Sci 257:1021-1032.

[16] Brandt M and Ruland W (1996) SAXS studies on the deformation of macro-lattices in block copolymers, Acta Polym 47:498-506.

[17] Wilke W and Bratrich M (1991) Investigation of the Superstructure of Polymers during Deformation by Synchrotron Radiation, J Appl Cryst 24:645-650.

[18] Grubb D and Bala V (1999) Simultaneous SAXS, WAXS and local strain measurement: 
deformation of row-structure in LLDPE, Polym Mater Sci Eng 81:357-358.

[19] Fu B X, Hsiao B S, Pagola S, Stephens P, White H, Rafailovich M, Sokolov J, Mather P T, Jeon H G, Phillips S, Lichtenhan J and Schwab J (2000) Structural development during deformation of polyurethane containing polyhedral oligomeric silsesquioxanes (POSS) molecules, Polymer 42:599-611.

[20] Yeh F, Hsiao B S, Sauer B B, Michel S and Siesler H W (2003) In-Situ Studies of Structure Development during Deformation of a Segmented Poly(urethane-urea) Elastomer, Macromolecules 36:1940-1954.

[21] Stribeck N, Polizzi S, Bösecke P and Zachmann H G (1989) Investigations of strained S-B-S block copolymers with dilute elastic networks, Rev Roumaine Chem 34:635-648.

[22] Stribeck N, Bösecke P and Polizzi S (1989) SAXS investigation on the influence of oil dilution on morphological changes in a SBS block copolymer during the first draw cycle, Colloid Polym Sci 267:687-701.

[23] Stribeck N (1999) The Equatorial Small-Angle Scattering During the Straining of Poly(ether ester) and its Analysis, J Polym Sci Part B Polym Phys 37:975-981.

[24] Stribeck N, Fakirov S and Sapoundjieva D (1999) Deformation Behavior of a Poly(ether ester) Copolymer. Quantitative Analysis of SAXS Fiber Patterns, Macromolecules 32:3368-3378.

[25] Fakirov S, Samokovlijsky O, Stribeck N, Apostolov A A, Denchev Z, Sapoundjieva D, Evstatiev M, Meyer A and Stamm M (2001) Nanostructure Deformation Behavior in Poly(ethylene terephthalate)/ Polyethylene Drawn blend as Revealed by Small-Angle Scattering of Synchrotron X Radiation, Macromolecules 34:3314-3317.

[26] Stribeck N, Buzdugan E, Ghioca P, Serban S and Gehrke R (2002) Nanostructure evolution of SIS thermoplastic elastomers during straining as revealed by USAXS and multidimensional chord distribution analysis, Macromol Chem Phys 203:636-644.

[27] Stribeck N, Androsch R and Funari S S (2003) Nanostructure Evolution of Homogeneous Poly(ethylene-co-1-octene) as a Function of Strain, Macromol Chem Phys 204:12021216.

[28] Stribeck N, Fakirov S, Apostolov A A, Denchev Z and Gehrke R (2003) Deformation Behavior of PET, PBT and PBT-Based Thermoplastic Elastomers as Revealed by SAXS from Synchrotron, Macromol Chem Phys 204:1000-1013.

[29] Stribeck N and Funari S S (2003) Nanostructure Evolution in a Poly(ether ester) Elastomer during Drawing and the Displacement of Hard Domains from Lamellae, J Polym Sci Part B Polym Phys 41:1947-1954.

[30] Sauer B B, McLean R S, Brill D J and Londono D J (2002) Norphology and Orientation during the Deformation of Segmented Elastomers Studied with Small-Angle X-ray Scattering and Atomic Force Microscopy, J Polym Sci Part B Polym Phys 40:1727-1740.

[31] Murakami S, Yamakawa M, Tsuji M and Kohjiya S (1996) Structure development in the uniaxial - drawing process of poly(ethylene naphthalate), Polymer 37:3945-3951.

[32] Séguéla R and Prud'homme J (1988) Affinity of Grain Deformation in Mesomorphic Block Polymers Submitted to Simple Elongation, Macromolecules 21:635-643.

[33] Lee H S, Yoo S R and Seo S W (1999) Domain and segmental deformation behavior of thermoplastic elastomers using synchrotron SAXS and FTIR methods, J Polym Sci Part B Polym Phys 37:3233-3245.

[34] Welsh G E, Blundell D J and Windle A H (2000) A transient mesophase on drawing polymers based on polyethylene terephthalate (PET) and polyethylene naphthoate (PEN), J Mater Sci 35:5225-5240.

[35] Hernández J J, García Gutiérrez M C, Nogales A, Rueda D R, Sanz A, Sics I, Hsiao B S, Roslaniec Z, Broza G and Ezquerra T A (2007) Deformation behaviour during cold drawing of nanocomposites based on single wall carbon nanotubes and poly(ether ester) 
copolymers, Polymer 48:3286-3293.

[36] Bras W, Mant G R, Derbyshire G E, O'Kane W J, Helsby W I, Hall C J and Ryan A J (1995) Real-Time Simultaneous Wide- and Small-Angle Fibre Diffraction, J Synchrotron Rad 2:87-92.

[37] Hughes D J, Mahendrasingam A, Martin C, Oatway W B, Heeley E L, Bingham S J and Fuller W (1999) An instrument for the collection of simultaneous small and wide angle $\mathrm{x}$-ray scattering and stress-strain data during deformation of polymers at high strain rates using synchrotron radiation sources, Rev Sci Instrum 70:4051-4054.

[38] Chen X, Yoon K, Burger C, Sics I, Fang D, Hsiao B S and Chu B (2005) In-situ Xray scattering studies of a unique toughening mechanism in surface-modified carbon nanofiber/UHMWPE nanocomposite films, Macromolecules 38:3883-3893.

[39] Hosemann R and Bagchi S N (1962) Direct Analysis of Diffraction by Matter, NorthHolland, Amsterdam.

[40] Guinier A (1963) X-Ray Diffraction, Freeman, San Francisco.

[41] Alexander L E (1979) X-Ray Diffraction Methods in Polymer Science, Wiley, New York.

[42] Feigin L A and Svergun D I (1987) Structure Analysis by Small-Angle X-Ray and Neutron Scattering, Plenum Press, New York.

[43] Baltá Calleja F J and Vonk C G (1989) X-Ray Scattering of Synthetic Polymers, Elsevier, Amsterdam.

[44] Stribeck N (2007) X-Ray Scattering of Soft Matter, Springer, Heidelberg, New York.

[45] Stribeck N (2001) Extraction of domain structure information from small-angle X-ray patterns of bulk materials, J Appl Cryst 34:496-503.

[46] Stribeck N and Fakirov S (2001) Three-Dimensional chord Distribution Function SAXS Analysis of the Strained Domain Structure of a Poly(ether ester) Thermoplastic Elastomer, Macromolecules 34:7758-7761.

[47] Ruland W (1977) The evaluation of the small-angle scattering of lamellar two-phase systems by means of interface distribution functions, Colloid Polym Sci 255:417-427.

[48] Ruland W (1978) The evaluation of the small-angle scattering of anisotropic lamellar twophase systems by means of interface distribution functions, Colloid Polym Sci 256:932936.

[49] Stribeck N and Ruland W (1978) Determination of the Interface Distribution Function of Lamellar Two-Phase Systems, J Appl Cryst 11:535-539.

[50] Méring J and Tchoubar-Vallat D (1965) X-Rays. Small-Angle X-Ray Scattering in diluted suspensions. Computations of chord distributions (fr.), C R Acad Sc Paris 261:3096-3099.

[51] Méring J and Tchoubar-Vallat D (1966) Solid State Physics. Diffuse Small-Angle X-Ray Scattering from concentrated systems (fr.), C R Acad Sc Paris 262:1703-1706.

[52] Méring $\mathbf{J}$ and Tchoubar D (1968) Interpretation of the SAXS from porous systems. Part I. (french), J Appl Cryst 1:153-165.

[53] Tchoubar D and Méring J (1969) Interpretation of the SAXS from porous systems. Part II. (french), J Appl Cryst 2:128-138.

[54] Glatter O and Kratky O, eds. (1982) Small Angle X-ray Scattering, Academic Press, London.

[55] Debye P and Menke H (1931) Study of molecular order in liquids by means of X-rays (ger.), Erg techn Rontgenkunde 2:1-22.

[56] Lengeler B, Schroer C G, Kuhlmann M, Benner B, Günzler T F, Kurapova O, Zontone F, Snigirev A and Snigireva I (2005) Refractive X-ray lenses, J Phys D Appl Phys 38:A218A222.

[57] Schroer C G, Kuhlmann M, Roth S V, Gehrke R, Stribeck N, Almendarez Camarillo A and Lengeler B (2006) Mapping the local nanostructure inside a specimen by tomographic small-angle x-ray scattering, Appl Phys Lett 88:164102. 
[58] Stribeck N, Almendarez Camarillo A, Nöchel U, Schroer C, Kuhlmann M, Roth S V, Gehrke R and Bayer R K (2006) Volume-Resolved Nanostructure Survey of a Polymer Part by Means of SAXS Microtomography, Macromol Chem Phys 207:1239-1249.

[59] Stribeck N, Downloads, http://www.chemie.uni-hamburg.de/tmc/stribeck/dl.

[60] Hammersley A P, FIT2D V12.012 Reference Manual, http://www.esrf.fr/computing/scientific/FIT2D/.

[61] Stribeck N, Nöchel U, Funari S S and Schubert T (2007) Comparison of SAXS Patterns Obtained by the Stretch-Hold Technique and by X-Raying During Continuous Stretching of Polypropylene, J Polym Sci Polym Phys :submitted.

[62] Stribeck N, Nöchel U, Funari S S, Schubert T and Timmann A (2007) Nanostructure Evolution in Polypropylene during a Load-Reversal Mechanical Test, Macromol Chem Phys :submitted.

[63] Putthanarat S, Stribeck N, Fossey S A, Eby R K and Adams W W (2000) Investigation of the nanofibril of silk fibers, Polymer 41:7735-7747.

[64] Müller M, Czihak C, Vogl G, Fratzl P, Schober H and Riekel C (1998) Direct Observation of Microfibril Arrangement in a Single Native Cellulose Fiber by Microbeam Small-Angle X-ray Scattering, Macromolecules 31:3953-3957.

[65] Reiterer A, Lichtenegger H, Fratzl P and Stanzl-Tschegg S E (2001) Deformation and energy absorption of wood cell walls with different nanostructure under tensile loading, $J$ Mater Sci 36:4681-4686.

[66] Stribeck N, Almendarez Camarillo A, Roth S V, Fakirov S and Bhattacharyya D (2005) Highly Oriented Nanofibrillar Polymer Blends as Models for USAXS Studies, Hasylab Ann Rep 1:897-898.

[67] Stribeck N, Fakirov S, Heinemanns K, Friedrich K, Almendarez Camarillo A, Bhattacharyya D and Roth S V (2005) USAXS Studies on Undrawn and Highly Drawn PP/PET Blends, Hasylab Ann Rep 1:997-998.

[68] Glatter O (1981) Convolution Square Root of Band-Limited Symmetrical Functions and its Application to Small-Angle Scattering Data, J Appl Cryst 14:101-108.

[69] Pedersen J S (1997) Analysis of small-angle scattering data from colloids and polymer solutions: modeling and least-squares fitting, Adv Coll Interf Sci 70:171-210.

[70] Damaschun G, Müller J J and Pürschel H V (1971) Desmearing of noisy small-angle X-ray scattering curves (Ger.), Acta Cryst A27:11-18.

[71] Riekel C, García Gutiérrez M C, Gourrier A and Roth S V (2003) Recent synchrotron radiation microdiffraction experiments on polymer and biopolymer fibers, Anal Bioanal Chem 376:594-601.

[72] Wang Y D and Cakmak M (2001) Spatial variation of structural hierarchy in injection molded PVDF and blends of PVDF with PMMA. Part II. Application of microbeam WAXS pole figure and SAXS techniques, Polymer 42:4233-4251.

[73] Blake F C (1933) On the Factors Affecting the Reflection Intensities by the Several Methods of X-Ray Analysis of Crystal Structures, Rev Mod Phys 5:169-202.

[74] Hendershot O P (1937) Absorption Factor for the Rotating Crystal Method of Crystal Analysis, Rev Sci Instr 8:324-326. 


\section{Contents}

1 Introduction 1

2 Scattering theory and materials structure 3

2.1 Relation Between a CDF and IDFs . . . . . . . . . . . . . 6

3 Analysis options derived from scattering theory 6

3.1 Completeness - a preliminary note $\ldots \ldots \ldots 6$

3.2 Analysis options . . . . . . . . . . . . . . . 7

3.3 Parameters, functions and operations . . . . . . . . . . . . 7

4 The experiment $\quad 8$

$4.1 \quad$ Principal design . . . . . . . . . . . . . . . 8

4.2 Engineering solutions $\ldots \ldots \ldots \ldots \ldots$

4.2.1 Setup for mechanical testing .............. 8

4.2.2 Setup for micro-tomography under bending load . . . . . . . . . 11

4.3 Scattering data and its evaluation . . . . . . . . . . . 11

5 Techniques: Dynamic vs. stretch-hold 15

6 Advanced goal: Identification of mechanisms 15

7 Observed promising effects from stretch-hold experiments 18

7.1 Orientation of nanofibrils in highly oriented polymer blends by means of USAXS 18

7.2 USAXS Studies on undrawn and highly drawn PP/PET blends $\ldots \ldots \ldots$

8 Choosing experiments $\quad 20$

8.1 Experiments with a macrobeam . . . . . . . . . . . 20

8.2 Experiments with a microbeam $\ldots \ldots \ldots \ldots \ldots$ 


\section{Index}

absorption, 3

absorption factor, 21

autocorrelation, 4

background, 14

Be-lens, 11

beam stop, 9

CDF, 8

chord distribution function, 8

chord length distribution, 5

Compton scattering, 3

continuous, see straining technique, dynamic

contrast, 5

correlation, 4

correlation function, $\mathbf{5}$

reduced, 7

deconvolution, 16, 20

density

jump, 6

desmearing, see deconvolution

dynamic, see straining technique

electron density, 4

elongation, 15

fluorescence, 3

Fourier transform, $\mathbf{3}$

Fraunhofer approximation, 3

ghost, $\mathbf{5}$

gradient, 5

harmonic

analysis, $\mathbf{4}$

function, 4

invariant, 7

scattering power, 7

irradiated volume, 7

kinematic scattering theory, 3

Laplacian, 5

marks, fiducial, 8

materials

multiphase, 5

micro-CT, 12

microbeam, 11 scanning, 21

multiple scattering, 3

operator

absolute square, 8

Patterson function, 4

polarization, 3

SAXS, 1, 2, 11

scattering

normalization, 21

scattering amplitude, 4, 7

slice

one-dimensional, 6

small-angle, see SAXS

step-wise, see straining technique, stretchhold

strain rate, $\mathbf{2}, 3$

straining technique

dynamic, 2, 15

stretch-hold, 1, 2, 3, 8, 15

stretch-hold, see straining technique

structure

longitudinal, 6

transversal, 6

symmetry

point, 4

ultra-small angle, see USAXS

USAXS, 8, 11

voxel, 11 